\title{
Synapsins Contribute to the Dynamic Spatial Organization of Synaptic Vesicles in an Activity-Dependent Manner
}

\author{
Eugenio F. Fornasiero, ${ }^{1}$ Andrea Raimondi, ${ }^{2}$ Fabrizia C. Guarnieri, ${ }^{1}$ Marta Orlando, ${ }^{2}$ Riccardo Fesce, ${ }^{1,3}$ \\ Fabio Benfenati, ${ }^{2,4}$ and Flavia Valtorta ${ }^{1}$ \\ ${ }^{1}$ San Raffaele Scientific Institute and Vita-Salute University, I-20132 Milan, Italy, ${ }^{2}$ Department of Neuroscience and Brain Technologies, Italian Institute of \\ Technology, I-16163 Genoa, Italy, ${ }^{3}$ Neuroscience Center, Insubria University, I-21100 Varese, Italy, and ${ }^{4}$ Department of Experimental Medicine, University \\ of Genoa and National Institute of Neuroscience, I-16132 Genoa, Italy
}

The precise subcellular organization of synaptic vesicles (SVs) at presynaptic sites allows for rapid and spatially restricted exocytotic release of neurotransmitter. The synapsins (Syns) are a family of presynaptic proteins that control the availability of SV s for exocytosis by reversibly tethering them to each other and to the actin cytoskeleton in a phosphorylation-dependent manner. Syn ablation leads to reduction in the density of SV proteins in nerve terminals and increased synaptic fatigue under high-frequency stimulation, accompanied by the development of an epileptic phenotype. We analyzed cultured neurons from wild-type and Syn I,II,III ${ }^{-/-}$triple knock-out (TKO) mice and found that SVs were severely dispersed in the absence of Syns. Vesicle dispersion did not affect the readily releasable pool of SVs, whereas the total number of SVs was considerably reduced at synapses of TKO mice. Interestingly, dispersion apparently involved exocytosis-competent SVs as well; it was not affected by stimulation but was reversed by chronic neuronal activity blockade. Altogether, these findings indicate that Syns are essential to maintain the dynamic structural organization of synapses and the size of the reserve pool of SVs during intense SV recycling, whereas an additional Syn-independent mechanism, whose molecular substrate remains to be clarified, targets SVs to synaptic boutons at rest and might be outpaced by activity.

\section{Introduction}

Neurotransmitter release is characterized by high speed and plasticity compared with other kinds of exocytosis. These features are made possible by its tight regulation through an array of proteinprotein interactions, as well as by the spatial and functional organization of the synapse. Thus, clusters of synaptic vesicles (SVs) concentrate at active zones (AZs), and only docked and primed SVs undergo fusion after a stimulus. In addition, various pools of SVs can be identified, based on their propensity to undergo exocytosis after distinct stimulation patterns (Valtorta and Benfenati, 1995; Murthy and De Camilli, 2003; Denker and Rizzoli, 2010).

The synapsins (Syns) represent a family of neuron-specific phosphoproteins (Kao et al., 1999) that in mammals are encoded

\footnotetext{
Received March 29, 2012; revised June 29, 2012; accepted July 12, 2012.

Author contributions: E.F.F. and F.V. designed research; E.F.F. and A.R. performed research; E.F.F., A.R., M.O., and R.F. analyzed data; E.F.F., F.C.G., R.F., F.B., and F.V. wrote the paper.

This work was supported by research grants from the Italian Ministry of University and Research (through Projects of Significant National Interest to F.B. and F.V.), the Society of San Paolo, Turin (F.B. and F.V.), and TelethonItaly Grant GGP09134 (F.B. and F.V.). We thank Drs. Serena Bellani and Davide Pozzi for assistance in preparing the manuscript, Anna Fassio for suggestions for the synaptopHluorin experiments, Luigi Naldini for providing the selfinactivating lentiviral vector, and Elena Monzani for technical assistance. ALEMBIC (an advanced microscopy laboratory established by the San Raffaele Scientific Institute and the Vita-Salute San Raffaele University) provided the facilities for performing part of the experiments. F.C.G. conducted this study as partial fulfillment for her PhD in Molecular Medicine, Program in Neuroscience, San Raffaele University, Milan, Italy.

The authors declare no competing financial interests.

Correspondence should be addressed to Flavia Valtorta, DIBIT 3A2, San Raffaele Scientific Institute, Via Olgettina 58,20132 Milan, Italy. E-mail: valtorta.flavia@hsr.it.

DOI:10.1523/JNEUROSCI.1554-12.2012

Copyright $\odot 2012$ the authors $\quad 0270-6474 / 12 / 3212214-14 \$ 15.00 / 0$
}

by three genes. Syns localize at nerve terminals, in which they interact with each other, with SVs and actin (for review, see Cesca et al., 2010). In mature neurons, Syns have been proposed to maintain a reserve pool of vesicles by tethering SVs to each other and to actin to control the availability of SVs for release through their phosphorylation-dependent dissociation from SVs and actin (Valtorta et al., 1992; Benfenati et al., 1993; Ceccaldi et al., 1995; Chi et al., 2001, 2003; Baldelli et al., 2007) and to play a role in the post-docking steps of exocytosis (Hilfiker et al., 1998, 2005; Humeau et al., 2001; Fassio et al., 2006; Hvalby et al., 2006; Sun et al., 2006; Baldelli et al., 2007, Chiappalone et al., 2009).

Deletion of single or multiple SYN genes (with the notable exception of SYN3; Feng et al., 2002) produces an epileptic phenotype (Li et al., 1995; Rosahl et al., 1995; Gitler et al., 2004; Boido et al., 2010; Ketzef et al., 2011), and mutations in SYN1 are associated with human epilepsy and/or autism (Garcia et al., 2004; Fassio et al., 2011). Neurons from single or multiple Syn knockout $(\mathrm{KO})$ mice exhibit impairments in inhibitory neurotransmission and enhancement in excitatory transmission, accompanied by alterations in synaptic plasticity (Rosahl et al., 1995; Gitler et al., 2004; Hvalby et al., 2006; Baldelli et al., 2007; Chiappalone et al., 2009; Farisello et al., 2012). In nerve terminals, a selective decrease in the density of SVs is observed (Li et al., 1995; Rosahl et al., 1995; Gitler et al., 2004; Siksou et al., 2007). In living animals, the decrease in SVs precedes the onset of epilepsy, raising the possibility that the decreased number of SVs has a causal role in epileptogenesis.

In search for the mechanisms underlying the alterations in synaptic transmission observed in the absence of Syns, we have 
examined the organization and mobility of SVs at nerve terminals of Syn I,II,III ${ }^{-1-}$ triple KO (TKO) mice and their dependence on neuronal activity. The results show that, in the absence of Syns, SVs acquire higher mobility and become dispersed along the axon. Prolonged inactivity of the synapse leads to reclustering of SVs at nerve terminals, indicating that molecules other than the Syns are able to target SVs at nerve terminals but that the Syns are essential for retaining SVs close to release sites during neuronal activity.

\section{Materials and Methods}

Materials. Rabbit antisera against vesicular glutamate transporter-1 (VGLUT1), vesicular GABA transporter (VGAT), Piccolo and monoclonal antibodies against Synaptotagmin I (Syt), Ras analogous in brain-3a (Rab3a), and vesicle-associated membrane protein 2 (VAMP2) were purchased from Synaptic Systems. The monoclonal antibody against postsynaptic density protein-95 (PSD-95) was purchased from Millipore. The monoclonal antibody against a Bassoon epitope was from Stressgen Bioreagents. The polyclonal anti-synaptophysin (Syp; G111) was raised in our laboratory (Valtorta et al., 1988). Tetramethylrhodamine isothiocyanate (TRITC)- and fluorescein isothiocyanate (FITC)-conjugated goat antirabbit and goat anti-mouse secondary antibodies were from Jackson ImmunoResearch. Citrate-free tetrodotoxin (TTX) was from Latoxan. All other reagents were purchased from standard commercial suppliers.

Mice. Homozygous Syn I,II,III ${ }^{-1-}$ (TKO) mice were kindly provided by Drs. H. T. Kao (Brown University, Providence, RI) and Paul Greengard (Rockefeller University, New York, NY). TKO mice were re-derived on a C57BL/6J background (Charles River) obtaining single and multiple KOs. For genotyping, DNA was extracted from tails and analyzed by PCR as described previously (Gitler et al., 2004). Mice were housed (five per cage) under constant temperature $\left(22 \pm 1^{\circ} \mathrm{C}\right)$ and humidity $(50 \%)$ conditions with a $12 \mathrm{~h}$ light/dark cycle and were provided with food and water ad libitum. All experiments involving animals followed protocols in accordance with the guidelines established by the European Communities Council and approved by the San Raffaele Institutional Animal Care and Use Committee (IACUC number 467) of San Raffaele Scientific Institute and the Italian Ministry of Health.

Plasmids, lentiviral constructs, and virus production. The construct encoding for the chimeric VAMP2-based vesicular reporter protein SynaptopHluorin (SpH; Miesenböck et al., 1998) was kindly provided by Prof. J. E. Rothman (Memorial Sloan-Kettering Cancer Center, New York, NY). The pSypHy-EGFP-N1 plasmid containing the cDNA of the chimeric vesicular reporter protein synaptophysin-pHluorin (SypHy; Granseth et al., 2006) was a kind gift from Prof. L. Lagnado (Medical Research Council Laboratory of Molecular Biology, Cambridge, UK).

The SypHy cDNA was inserted into the self-inactivating lentiviral vector 282.pCCLsin.PPT.hPGK.deltaNGFRpre kindly provided by Prof. L. Naldini (Telethon Institute for Gene Therapy, San Raffaele Scientific Institute, Milan, Italy). Briefly, the pSypHy-EGFP-N1 was linearized with the restriction enzyme XhoI [New England Biolabs (NEB)]. The 5' protruding ends were blunted with the Quick Blunting kit (NEB). The SypHy cDNA was excised with the restriction enzyme XbaI (NEB). The 282.pCCLsin.PPT.hPGK.deltaNGFRpre vector was digested with the restriction enzymes SmaI and XbaI (NEB). Ligation was performed with the Quick Ligation kit (NEB). The lentiviral construct sequences were verified by automated sequencing at the PRIMM Facility (Milan, Italy). The lentiviral construct encoding Syp-enhanced yellow fluorescent protein (eYFP) has been described previously (Bonanomi et al., 2005). Viral stocks were prepared as described previously (Amendola et al., 2005) by transient cotransfection of HEK293T cells using the $\mathrm{Ca}^{2+}$-phosphate precipitation method with the transfer vector, the $\mathrm{pCMV} \Delta \mathrm{R} 8.74$ plasmid encoding Gag, Pol, Tat, and Rev, and the pMDG plasmid encoding the G glycoprotein of the vesicular stomatitis virus. The culture medium containing viral particles was harvested at 48 and $72 \mathrm{~h}$ after transfection, and viral preparations were concentrated by ultracentrifugation to increase titer. Viral titers (infectious particles) were determined by transduction of HEK293T cells with serial dilution of the viral stocks and evaluation of transduction efficiency by flow cytometry scoring of fluorescent cells.
Cell culture procedures. Astroglial cell cultures were prepared from brains of Sprague Dawley rats (Charles River) at postnatal day 2 as described previously (Kaech and Banker, 2006). Briefly, rat pups of either sex were killed by decapitation, brains were extracted from the skull, and then cerebellum, meninges, and all subcortical structures were removed to leave only the cerebral hemispheres. The tissue was minced with a blade into small pieces, washed with HBSS (Invitrogen) to remove the smallest debris, and then trypsinized in a solution containing $2.5 \%$ tryp$\sin$ (Sigma-Aldrich) and $1 \mathrm{mg} / \mathrm{ml}$ DNase (Sigma-Aldrich) for $15 \mathrm{~min}$ at $37^{\circ} \mathrm{C}$. After the incubation, cells were washed three times with HBSS and then dissociated. Cells were plated in modified Eagle medium (MEM) containing 10\% horse serum (Invitrogen), $0.6 \%$ glucose (Sigma-Aldrich), $2 \mathrm{~mm}$ glutamine (Lonza), $100 \mathrm{U} / \mathrm{ml}$ penicillin, and $100 \mu \mathrm{g} / \mathrm{ml}$ streptomycin (Lonza) and maintained at $37^{\circ} \mathrm{C}$ in $5 \% \mathrm{CO}_{2}$ humidified atmosphere. One day before preparing hippocampal cultures, the glial medium was removed and replaced with hippocampal medium [MEM supplemented with $1 \% \mathrm{~N} 2$ supplement (Invitrogen), 2 mm glutamine, $1 \mathrm{~mm}$ sodium pyruvate (SigmaAldrich), and $4 \mathrm{~mm}$ glucose].

Primary neuronal cultures were prepared from the hippocampi of embryonic day 17.5 embryos from wild-type (WT) and TKO mice of either sex as described previously (Banker and Cowan, 1977). The pregnant mouse was killed with $\mathrm{CO}_{2}$, and embryos were extracted and decapitated. Skulls were opened, and brains were dissected out and placed into HBSS. Hippocampi were removed under a dissecting microscope and collected. After $15 \mathrm{~min}$ of incubation with $0.25 \%$ trypsin in $\mathrm{HBSS}$ at $37^{\circ} \mathrm{C}$, the whole hippocampi were washed with HBSS to remove trypsin and then mechanically dissociated. Neurons stained with vital dye (Trypan blue; Sigma-Aldrich) were counted by using a Burker chamber. Neurons were plated on poly-L-lysine $(0.1 \mathrm{mg} /$ $\mathrm{ml}$; Sigma-Aldrich)-treated $24 \mathrm{~mm}$ glass coverslips at a density of 100,000 cells per coverslip (low-density cultures). Cells were plated in plating medium (MEM supplemented with $10 \%$ horse serum, $3.3 \mathrm{~mm}$ glucose, and 2 mM glutamine) and incubated for $1-4 \mathrm{~h}$ at $37^{\circ} \mathrm{C}$ in a $5 \% \mathrm{CO}_{2}$ humidified atmosphere to allow adhesion to the substrate. After plating, coverslips were transferred into the cell culture dish with a glia monolayer prepared as described above, containing hippocampal medium conditioned for at least $24 \mathrm{~h}$. Coverslips were turned upside down, with neurons facing the glia, separated by paraffin dots. To obtain homogeneous conditions for comparisons among the various genotypes, neurons used in the same experiment were prepared concomitantly. All experiments were performed in mature neurons [ $>15 \mathrm{~d}$ in vitro (DIV)].

For activity blockade experiments, TTX was resuspended in $10 \mathrm{~mm}$ sodium acetate, $\mathrm{pH} 4.5$, and used at a final concentration of $1 \mu \mathrm{M}$ for $48 \mathrm{~h}$ in neurons $>15$ DIV.

Transfection and viral transduction procedures. Transfections were performed with Lipofectamine 2000 (Invitrogen), according to the instructions of the manufacturer. Neurons at 14 DIV were placed in a clean dish and incubated for $1 \mathrm{~h}$ with hippocampal medium containing Lipofectamine and DNA constructs (in a 2:1 ratio) and then returned to the original dishes and analyzed at 18 DIV.

For infection, coverslips were placed in a clean dish containing gliaconditioned medium (MEM supplemented with 1\% N2 supplement, 2 mu glutamine, $1 \mathrm{~mm}$ sodium pyruvate, and $4 \mathrm{~mm}$ glucose; incubated for $10-15 \mathrm{~h}$ at $37^{\circ} \mathrm{C}$ in a $5 \% \mathrm{CO}_{2}$ humidified atmosphere in the presence of viral supernatant at $1-10$ multiplicity of infection). After transduction, neurons were returned to the original dishes and maintained in culture in glia-conditioned medium.

Cell labeling protocols. Standard immunofluorescence experiments were performed as described previously (Pennuto et al., 2003). Briefly, cells were rinsed once with Krebs-Ringer's solution (KRH)-EGTA (in mM: $130 \mathrm{NaCl}$, $5 \mathrm{KCl}, 1.2 \mathrm{KH}_{2} \mathrm{PO}_{4}, 1.2 \mathrm{MgSO}_{4}, 2 \mathrm{MgCl}_{2}, 2$ EGTA, $25 \mathrm{HEPES}$, and 6 glucose, $\mathrm{pH}$ 7.4), fixed for $15 \mathrm{~min}$ with $4 \%$ paraformaldehyde, $4 \%$ sucrose in $120 \mathrm{~mm}$ sodium phosphate buffer, $\mathrm{pH} 7.4$, supplemented with 2 mM EGTA. Mature cultures were always fixed in the presence of EGTA to prevent depolarization. Coverslips were rinsed three times with PBS and then incubated overnight at $4^{\circ} \mathrm{C}$ in a humidified chamber with the primary monoclonal antibody appropriately diluted in goat serum dilution buffer (GSDB; $15 \%$ goat serum, $450 \mathrm{~mm} \mathrm{NaCl}, 0.3 \%$ Triton X-100, and $20 \mathrm{~mm}$ sodium phosphate buffer, $\mathrm{pH}$ 7.4). For double immunofluorescence, the following day, cells were washed three times within 30 min with PBS and incubated with the polyclonal pri- 
a
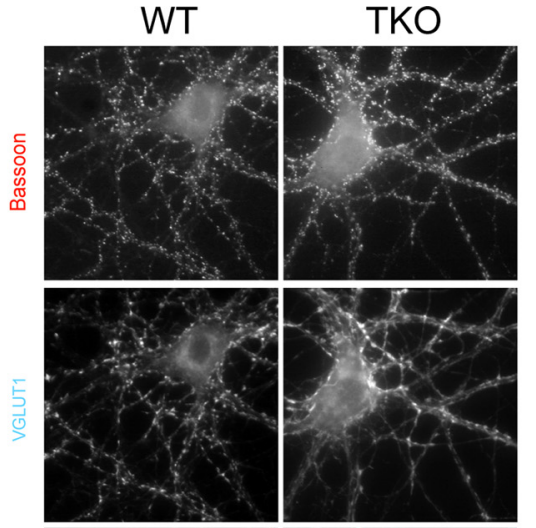

है

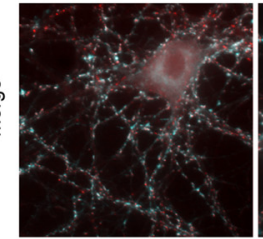

C

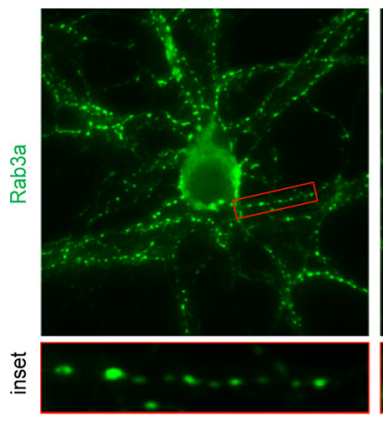

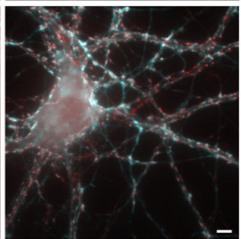

TKO

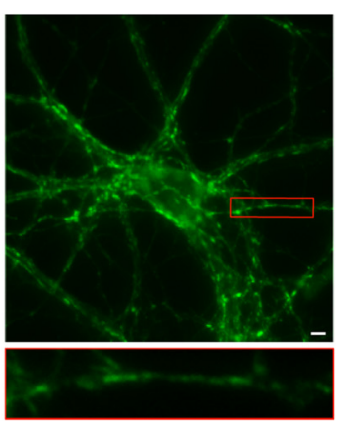

b
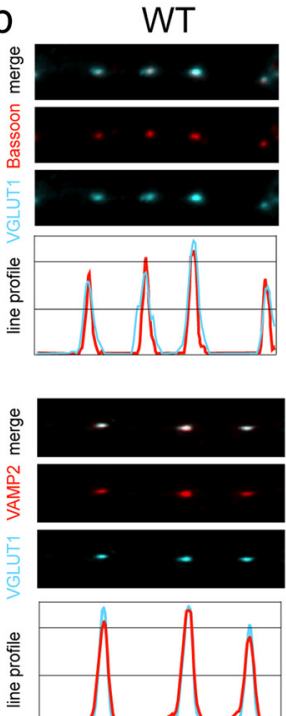

d
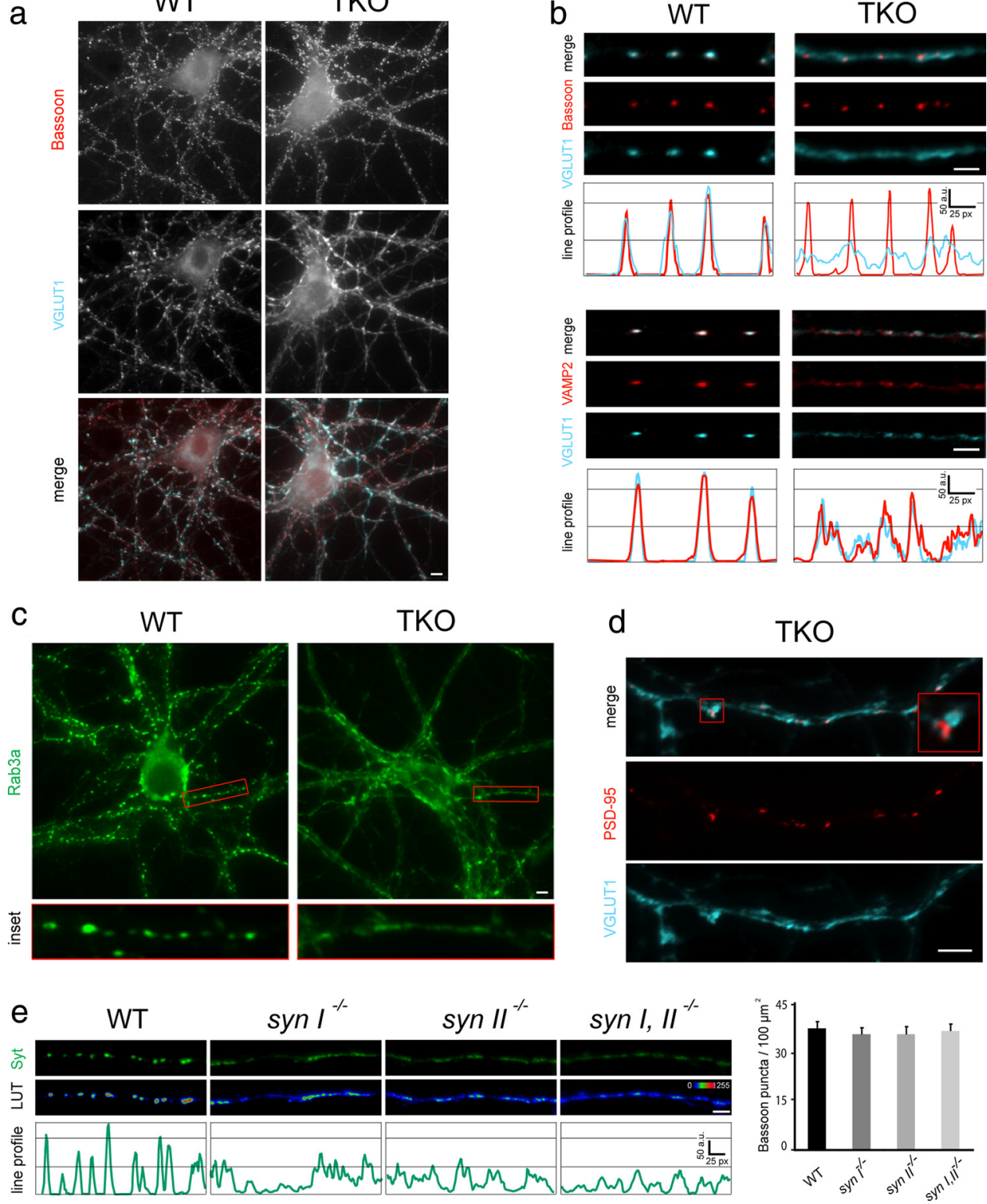

Figure 1. In Syn TKO hippocampal neurons, SV markers are dispersed along the axon. $\boldsymbol{a}$, Low-magnification images showing the distribution of immunofluorescence for the VGLUT1 and the presynaptic scaffolding protein Bassoon in WT and TKO neurons. $\boldsymbol{b}$, Fluorescence immunostaining of isolated axonal tracts of WT and TKO neurons and respective intensity line profiles. Top, One SV marker (VGLUT1) and one presynaptic marker (Bassoon); bottom, two SV markers (VGLUT1 and VAMP2). c, Immunofluorescence staining for the SV-associated protein Rab3a in WT and TK0 neurons and detail of isolated axonal tracts (insets). $\boldsymbol{d}$, Double immunofluorescence of SV (VGLUT1) and postsynaptic (PSD-95) markers in TKO neurons. The enlarged detail of a synapse is shown in the red box. $\boldsymbol{e}$, Left, Representative isolated axonal tracts of cultured neurons (>14 DIVs) from WT, Syn $I^{-/-}$, Syn $I^{-/-}$, and Syn I, II ${ }^{-/-}$mice showing the distribution pattern of immunofluorescence for the SV protein Syt. The corresponding lookup table (LUT) and the fluorescence intensity line profiles are reported under each image to appreciate longitudinal dispersion. Right, The average density of presynaptic puncta (visualized by immunofluorescence for Bassoon) is unchanged in these cultures (mean \pm SEM; $n=3$ independent preparations). Scale bars, $3 \mu \mathrm{m}$.

mary antibody for $2 \mathrm{~h}$ at room temperature (RT) in GSDB. Specimens were then washed three times within 30 min with PBS and incubated with the appropriate secondary antibodies for $90 \mathrm{~min}$ at RT. After three washes with PBS for 30 min, coverslips were mounted with 70\% glycerol in PBS supplemented with phenylenediamine $(1 \mathrm{mg} / \mathrm{ml})$ as an anti-bleaching agent. For retrospective immunofluorescence, all samples were fixed immediately after imaging. A circle was traced on the bottom coverslip using a diamond-tip scribing objective (Carl Zeiss) and used as a reference to relocate previously imaged fields. All the reagents used were from Sigma-Aldrich.

Electrical field stimulation. Stimulation was performed on SypHy- or SypeYFP-infected neurons with a Stimulus Isolated Unit (SIU-102; Warner Instruments) controlled by a stimulus generator (STG4004; Multichannel Systems) in a field stimulation chamber (Slotted Bath Chambers RC-46SLP; Warner Instruments). Coverslips were mounted on the stimulation chamber and incubated with $200 \mu \mathrm{l}$ of $\mathrm{KRH}$ (in mM: $130 \mathrm{NaCl}, 5 \mathrm{KCl}, 1.2 \mathrm{KH}_{2} \mathrm{PO}_{4}$,
1.2 $\mathrm{MgSO}_{4}, 2 \mathrm{CaCl}_{2}, 25$ HEPES, and 6 glucose, $\mathrm{pH}$ 7.4) supplemented with $10 \mu \mathrm{M}$ 6-cyano-7-nitroquinoxaline-2,3-dione (CNQX) and $50 \mu \mathrm{M} 2$-amino5-phosphonopentanoic acid (APV; Tocris Bioscience). After stimulation, KRH was substituted with $\mathrm{KRH}-\mathrm{NH}_{4} \mathrm{Cl}$ (in mM: $50 \mathrm{NH}_{4} \mathrm{Cl}, 80 \mathrm{NaCl}, 5 \mathrm{KCl}$, $1.2 \mathrm{KH}_{2} \mathrm{PO}_{4}, 1.2 \mathrm{MgSO}_{4}, 2 \mathrm{CaCl}_{2}, 25$ HEPES, and 6 glucose, $\mathrm{pH} 7.4$ ) to evaluate total vesicles.

Video microscopy and image analysis. Epifluorescence images were acquired with an inverted microscope (Axiovert 135; Carl Zeiss) equipped with epifluorescence $63 \times$ optics. Images were recorded with a C4742-98 ORCA II cooled charge-coupled device camera. Custom-made and built-in macros for NIH ImageJ were used for pseudo-coloring, line profile analysis, and kymograph production. When analyzed, all images from the same experiment were taken with the same settings.

To evaluate the distribution of SVs with respect to the AZ, epitopes were labeled with rabbit polyclonal or mouse monoclonal antibodies, 

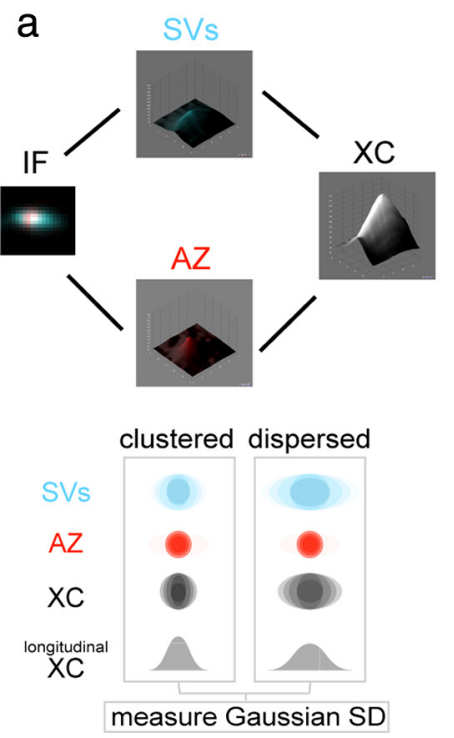

Figure 2. The dispersed SV markers represent intact SVs and are present in both excitatory and inhibitory neurons. $\boldsymbol{a}$, Illustration of how dispersion is quantified by signal cross-correlation analysis. Immunofluorescence images (IF) of fixed hippocampal neurons, double stained for SV markers and AZ proteins. Immunofluorescence images were acquired at constant settings. The cross-correlation surface curve for $4 \mu \mathrm{m}^{2}$ images was calculated (XC). An ellipsoidal Gaussian surface was fitted to XC, and the transversal and longitudinal SDs were computed. The bottom schematics represent the results of cross-correlation for clustered and dispersed SV proteins. The longitudinal projection is also represented (longitudinal XC). Higher values of Gaussian distribution indicate higher dispersion. $\boldsymbol{b}$, Results from the statistical analysis of longitudinal cross-correlation for presynaptic scaffolding proteins (either Bassoon or Piccolo) with excitatory (VGLUT1), inhibitory (VGAT), or shared (Syt) vesicular markers. Average from three independent experiments ( $>40$ boutons per condition) are reported (mean \pm SEM; ${ }^{* *} p<0.01$, Student's $t$ test vs WT).

followed by TRIC-conjugated (red) or FITC-conjugated goat anti-rabbit or anti-mouse secondary antibodies (shown in cyan). The cyan and red images were compared, the cross-correlation function was computed, and a set of parameters were recorded for each identified synapse with custom-made MATLAB (MathWorks) routines. These parameters included the following: (1) overall FITC/TRITC signal ratio; (2) longitudinal and transversal shift (translational offset, usually zero); (3) longitudinal and transversal width of SV dispersion (SD of the Gaussian bells describing the cross-correlation along the two axes); and (4) longitudinal and transversal asymptotic values. From these parameters, an idealized average crosscorrelation function was computed.

The axonal surface fluorescence and the localization of SV proteins were evaluated with $\mathrm{SpH}$ according to Sankaranarayanan et al. (2000). In detail, presynaptic puncta were defined as spots with $\Delta F$ ratios during acidification significantly higher $(>5$ SDs) than the background and immobile for $>4 \mathrm{~min}$. A region of interest (ROI) of $3 \times 3$ pixels $(2 \times$ binning) was positioned at the center of the peak (synaptic bouton), and an adjacent ROI of the same size was selected on the axon (extrasynaptic region). The levels of expression of the exogenous protein were verified during $\mathrm{NH}_{4} \mathrm{Cl}$ dequenching (total fluorescence) and confirmed by post hoc analysis (data not shown). The surface fluorescence was calculated in the synaptic regions as $\left[F_{0}-\left(\Delta F_{\mathrm{NH} 4 \mathrm{Cl}} / 19.7\right)\right]$, following Equation 7 of Sankaranaryanan et al. (2000). The values obtained in $\mathrm{KO}$ neurons were normalized to those of WT neurons. The amount of synaptic and extrasynaptic acidic vesicles is expressed as the average $\Delta F$ normalized for background correction.

For SypHy experiments, responding synapses were identified by highlighting the stimulus-evoked increase in SypHy fluorescence, and ROIs were then matched to exocytotic hotspots. The ROI size was $3 \times 3$ pixels at $2 \times$ binning $\left(0.42 \mu \mathrm{m}^{2}\right)$, and the precise location was automatically selected and computed through a custom-made NIH ImageJ macro based on the "Time Series Analyzer" ImageJ plugin (available at http://rsbweb.nih.gov/ij/plugins/time-series.html).

To estimate SV mobility in excitatory versus inhibitory neurons, SypeYFP fluorescence was monitored over time. Increases in SV mobility should produce greater variability of SV-related signals with time. Excit- atory and inhibitory neurons were infected with Syp-eYFP, and terminals were time-lapse imaged on an Axiovert 135 inverted microscope equipped with epifluorescence optics. To avoid artifacts attributable to sample mechanical drift, samples were habituated on a heat stage for $15 \mathrm{~min}$ before each experiment. Images were taken at $0.5 \mathrm{~Hz}$ for $120 \mathrm{~s}$. After each experiment, the positivity for either VGLUT1 or VGAT was assessed by retrospective immunofluorescence. Images were automatically aligned and bleach-corrected with custommade NIH ImageJ macros. ROIs of $3 \times 3$ pixels were selected on either excitatory or inhibitory synapses, and the average fluorescence and coefficient of variation (CV) (SD/mean) were calculated for both genotypes.

For time-lapse confocal observation of live cells expressing fluorescent constructs and fluorescence recovery after photobleaching (FRAP) experiments, images were acquired at $37^{\circ} \mathrm{C}$ in $\mathrm{KRH}$ on a Leica TCS SP2 AOBS confocal microscope with a $63 \times / 1.4$ NA oilimmersion objective, $5 \times$ zoom $(512 \times 512$ pixels). For FRAP analysis, three to five images were acquired with $5 \times$ zoom to define the prebleaching plateau with the single $488 \mathrm{~nm}$ laser line (520-650 nm detection). Bleaching was performed with $4-10$ pulses using the $488 \mathrm{~nm}$ and the $514 \mathrm{~nm}$ lines of an argon laser $(100 \mathrm{~mW}$ nominal output) at $90 \%$ power on designed areas. Fluorescence recovery was monitored by collecting either single-section images at $1 \mathrm{~s}$ intervals for fast bleaching trials or four image stacks (total $z=2 \mu \mathrm{m}$ ) for long bleaching trials. Images were corrected for drift with NIH ImageJ. For quantitative analysis of fluorescence recovery, data were double normalized as described previously (Phair et al., 2004). Double exponential fits of FRAP curves were obtained by minimizing square errors on average curves, and SEs of the fit coefficients were computed from the covariance matrix, using MATLAB software environment (MathWorks).

Transmission electron microscopy. Primary hippocampal neurons were fixed with $1.3 \%$ glutaraldehyde in $66 \mathrm{~mm}$ sodium cacodylate buffer, $\mathrm{pH}$ 7.4, postfixed in $1 \% \mathrm{OsO}_{4}, 1.5 \% \mathrm{~K}_{4} \mathrm{Fe}(\mathrm{CN})_{6}$, and $0.1 \mathrm{M}$ sodium cacodylate, en bloc stained with $0.5 \%$ uranyl acetate, dehydrated, and embedded in Epon. Ultrathin sections were contrasted with 2\% uranyl acetate and Sato's lead solution and observed with a JEOL JEM-1011 microscope at $100 \mathrm{kV}$, and images were taken with an ORIUS SC1000 CCD camera (Gatan). Morphometrical analysis of cross-section synaptic areas, AZ lengths, and total and docked SVs was done in NIH ImageJ. For the analysis of the spatial distribution of SVs, the position of each SV was located in NIH ImageJ, and the coordinates were transferred to CrimeStat (Ned Levine and Associates) in which we calculated the distance from the closest vesicle [mean nearest neighbor distance (MNND)] and the number of clustered vesicles (considered as a group of at least 8 SVs no more than $100 \mathrm{~nm}$ apart). For serial section analysis, ribbons of $60 \mathrm{~nm}$ ultrathin sections were collected on carbon-coated copper slot grids and imaged with a JEOL JEM-1011 microscope at $100 \mathrm{kV}$. The serial section images were aligned using the "MIDAS" and "align" programs of IMOD package (University of Colorado, Boulder, CO), and then both the SVs present in the synaptic bouton and those located along the axon (up to a maximum of $5 \mu \mathrm{m}$ from the selected bouton or until the next synaptic contact) were counted. Three-dimensional (3D) visualization of the SV distribution along the axon was done by segmenting the serial sections in IMOD.

For the evaluation of horseradish peroxidase (HRP) uptake, hippocampal neurons were electrically stimulated at 15 DIV in the presence of $10 \mu \mathrm{M} \mathrm{CNQX} / 50 \mu \mathrm{M}$ APV (Tocris Bioscience) and $10 \mathrm{mg} / \mathrm{ml} \mathrm{HRP}$ (Sigma), by applying 900 action potentials (APs) at $20 \mathrm{~Hz}$ in the field 
a
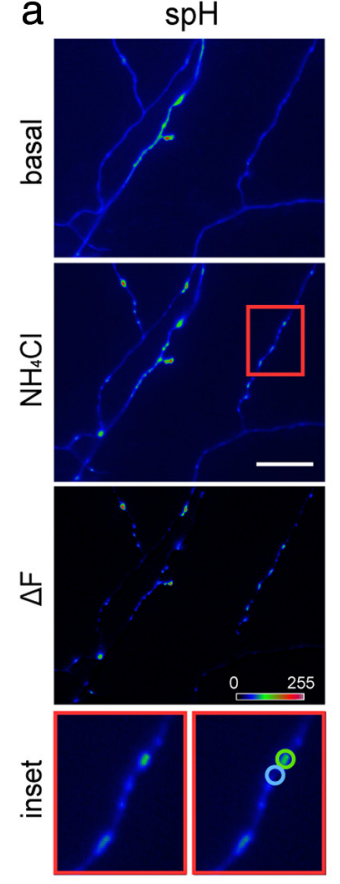

synaptic bouton

O extrasynaptic reqion b

C

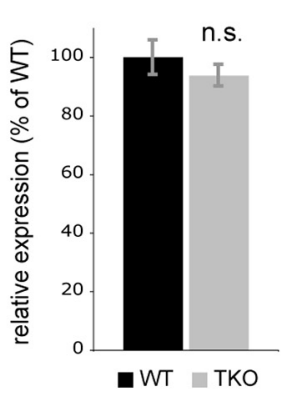

d synaptic

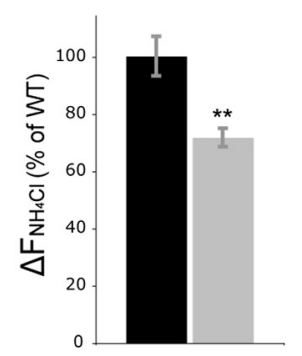
$\mathrm{SpH}$ expression acidic vesicles surface $\mathrm{SpH}$

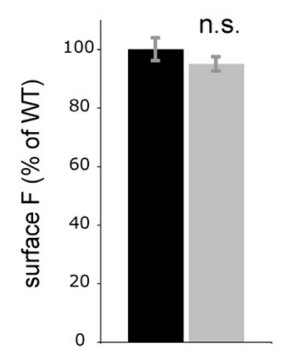

e

extrasynaptic acidic vesicles

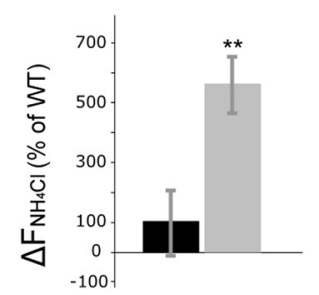

f Surface fluorescence (SypHy)

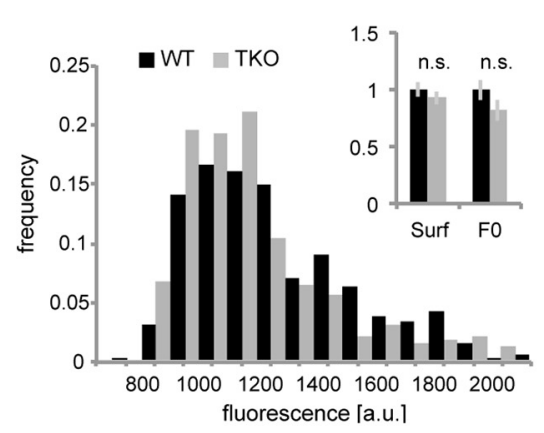

$\mathrm{h}$

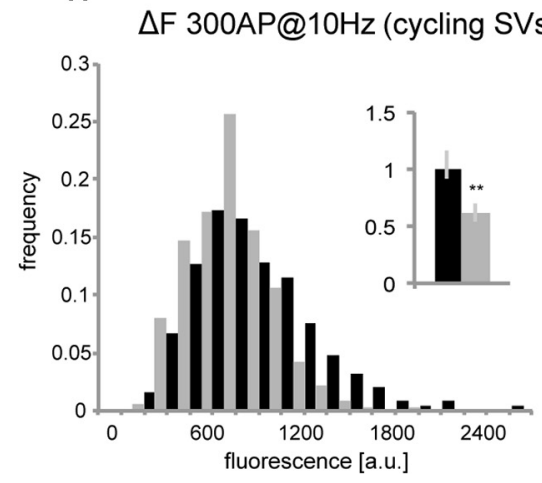

g $\quad \mathrm{F}$ 40AP@20Hz (RRP)

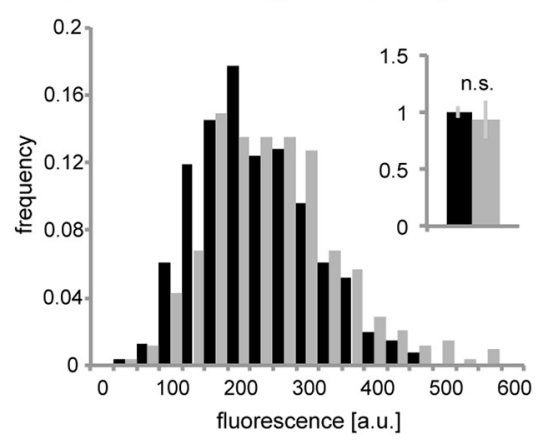

i

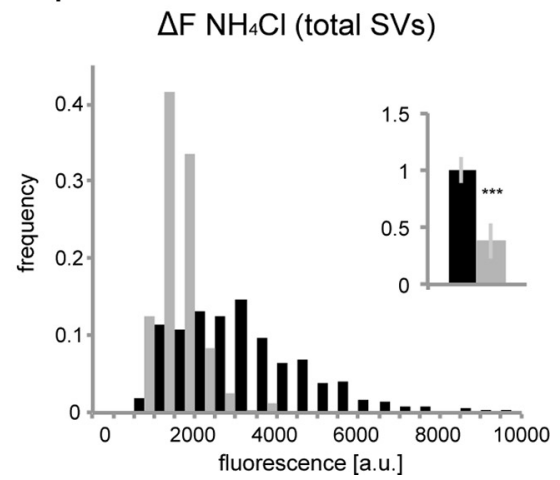

Figure 3. Dispersion of SV proteins in TKO neurons occurs in vesicular compartments and affects the recycling pool of SVs. Live WT and TKO hippocampal neurons were either transfected with SpH $(\boldsymbol{a}-\boldsymbol{e})$ or infected with SypHy $(\boldsymbol{f}-\boldsymbol{i})$ as fluorescent reporters of SV protein localization and SV exocytosis. $\boldsymbol{a}$, A representative SpH experiment is shown. Images from neurons overexpressing SpH were taken under basal conditions and after $\mathrm{NH}_{4} \mathrm{Cl}$ dequenching. In the inset, representative selections for image analysis are shown, comprising the synaptic area (at the bouton) and the adjacent extrasynaptic region. Quantification of the relative expression of $\mathrm{SpH}\left(\right.$ field $\left.\mathrm{F}_{\mathrm{NH} 4 \mathrm{Cl}}\right)(\boldsymbol{b})$, surface fluorescence of SpH at the terminal $\left[F_{0}-\left(\Delta F_{\mathrm{NH} 4 \mathrm{Cl}} / 19.7\right)\right](\boldsymbol{c})$, and estimate of acidic vesicles localized at either synaptic $(\boldsymbol{d})$ or extrasynaptic $(\boldsymbol{e})$ regions (for details, see Materials and Methods). Average fluorescence variation of $\mathrm{SpH}\left(\Delta F_{\mathrm{NH} 4 \mathrm{Cl}}\right)$ from three independent experiments ( $>200$ boutons per condition) is reported (mean $\pm \mathrm{SEM}$; ${ }^{* *} p<0.01$, Student's $t$ test vs WT). $\boldsymbol{f}-\boldsymbol{i}$, Evaluation of SV pools at hippocampal boutons of neurons infected with SypHy. $\boldsymbol{f}$, Frequency distribution of surface fluorescence (Surf) of SypHy at the terminal (data from all experiments are plotted). In the inset, the average surface stimulation chamber. Neurons were then washed and fixed with $1.3 \%$ glutaraldehyde in 66 mm sodium cacodylate buffer, pH 7.4. Neurons were incubated in the presence of 0.3

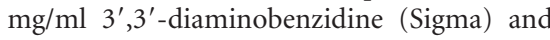
$0.03 \% \mathrm{H}_{2} \mathrm{O}_{2}$ up to $30 \mathrm{~min}$ and further processed as for conventional transmission electron microscopy (EM).

Data analyses and image composition. Data were analyzed using Microsoft Excel and Prism 5.0 software (GraphPad Software). Student's $t$ test was used for paired comparison, and $p$ values $<0.05$ were considered significant. For multiple comparisons, one-way ANOVA followed by Tukey's multiple comparison test was used. When data were not normally distributed, the Mann-Whitney-Wilcoxon test was used for paired comparisons. Image composition and drawing was performed with NIH ImageJ and the Adobe Creative Suite2 (Adobe Systems).

\section{Results}

Enhanced dispersion of SV components at presynaptic boutons lacking Syns Primary hippocampal neurons were prepared from WT or TKO embryos and maintained in culture for at least 15 DIV. The general appearance of these cultures was normal (Fig. 1a). Nevertheless, a more detailed observation of the distribution of the SV glutamate transporter VGLUT1 and the presynaptic scaffolding marker Bassoon on isolated axonal tracts revealed that, in contrast to the typical punctate pattern of staining observed in WT neurons, the staining was more diffuse in the intersynaptic regions along the axon of TKO neurons (Fig. 1b, top). Staining for the presynaptic AZ marker Bassoon was punctate in both WT and TKO neurons and, in the case of WT neurons, the distribution of Bassoon fluorescence precisely overlapped that of VGLUT1. We performed additional colocalization studies to test whether dispersion occurred independently for various SV proteins. For all pairs of SV proteins analyzed (VGLUT1/Syt; VGAT/ Syt; VGLUT1/VAMP2; VGAT/VAMP2; Syp/Syt), staining was essentially superimposable in both WT and TKO neurons, suggesting that dispersion affected intact

fluorescence and the $F_{0}$ from four independent experiments normalized to the WT values are shown. $\boldsymbol{g}, \boldsymbol{h}$, A short stimulation protocol ( $40 \mathrm{APs}$ at $20 \mathrm{~Hz}$ ) was used to estimate the readily releasable pool of vesicles (RRP), whereas a longer stimulation protocol ( $300 \mathrm{APs}$ at $10 \mathrm{~Hz}$ ) was used to quantify the recycling pool of SVs at synapses. $i$, Dequenching of SypHy by application of $\mathrm{NH}_{4} \mathrm{Cl}$ was used as control of total $\mathrm{SV}$ s at boutons. In the insets $(\boldsymbol{g}-\boldsymbol{i})$, data from more than four independent experiments ( $>500$ boutons per condition) are reported (mean \pm SEM; ${ }^{* *} p<0.01$, Student's $t$ test vs WT). Scale bar, $5 \mu \mathrm{m}$. 
a
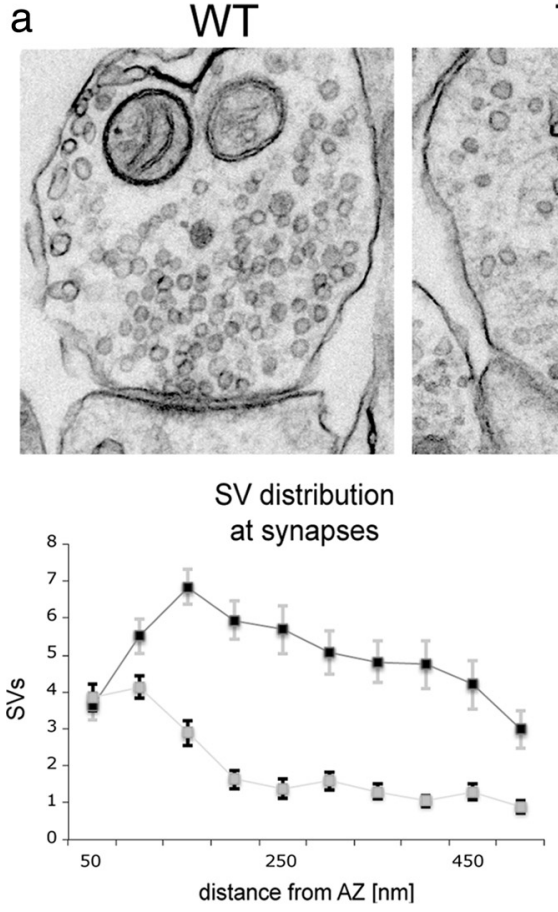

b
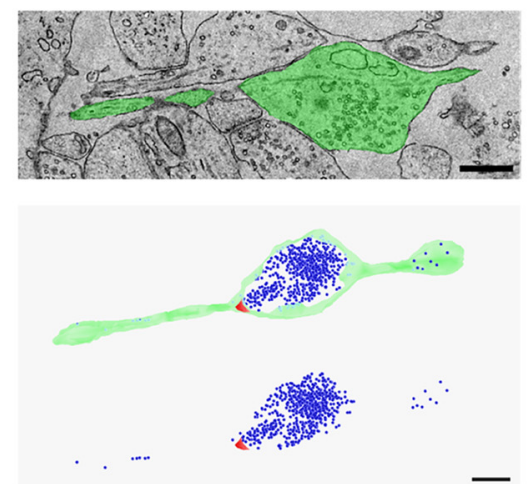

axonal SVs

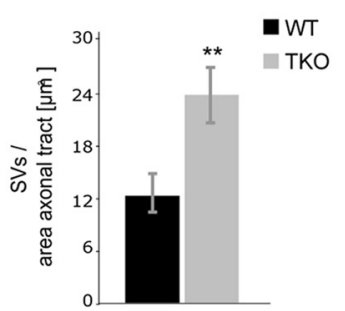

synaptic SVs / axonal SVs

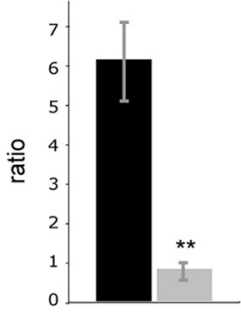

C

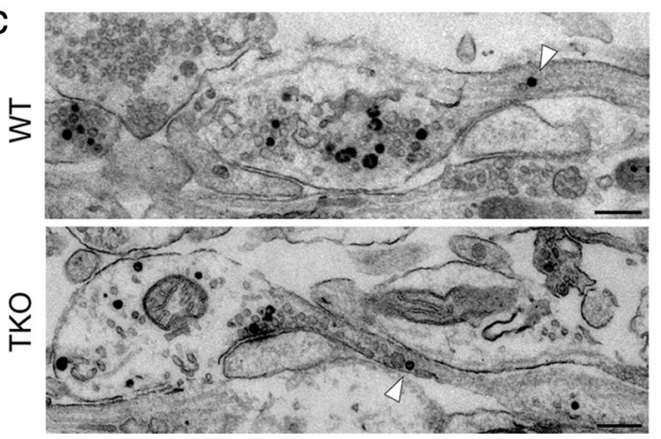

TKO

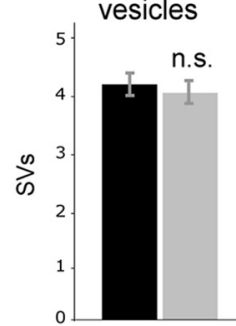

- WT

- TKO

SV density

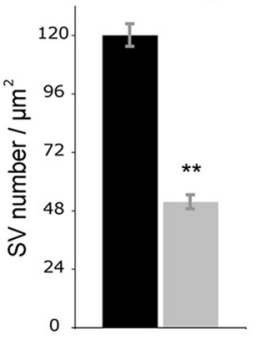

MNND

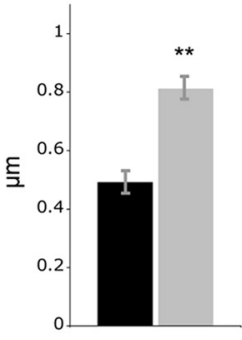

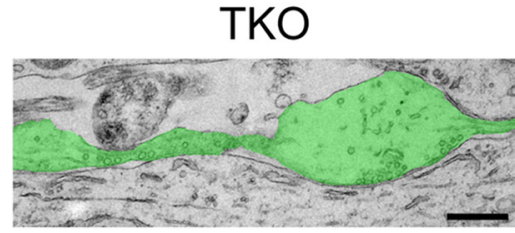

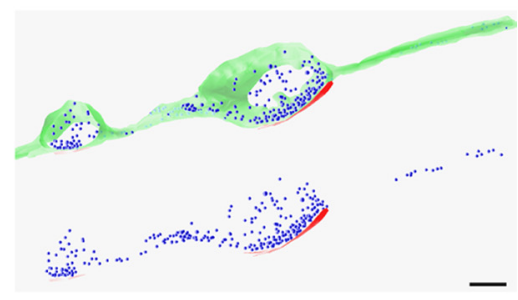

synaptic area / axonal area

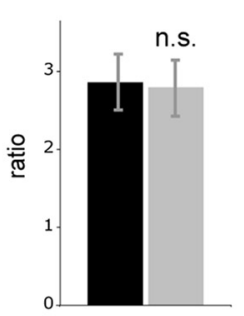

$\mathrm{HRP}^{+} \mathrm{SV}$ distribution

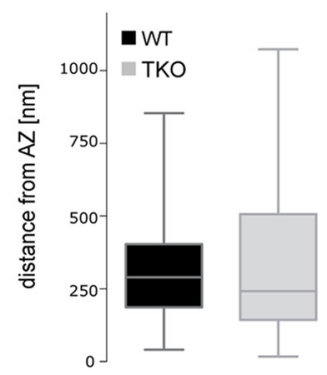

Figure 4. Evaluation of SV dispersion in WT and TKO terminals by EM. $\boldsymbol{a}$, Representative EM images of WT and TKO synapses and morphometric analysis of SV density, SV distribution (as distance from the AZ), and number of SVs docked at the AZ and SV
SVs, rather than isolated vesicle components (Fig. $1 b$, bottom and data not shown). Indeed, also proteins reversibly associated with the SV membrane had a diffused distribution along the axons of TKO neurons (Fig. 1c). Conversely, the strictly punctate staining observed for PSD-95 indicated that postsynaptic density organization is preserved, similar to presynaptic AZs, in TKO neurons (Fig. 1d).

Notably, dispersion of SVs was also observed in neurons from Syn I-/-, Syn $I I^{-/-}$, and Syn $I, I^{-/-}$mice, suggesting that deletion of either Syn isoform is sufficient to generate this phenotype (Fig. 1e).

Because presynaptic scaffolding appears to be unaltered in TKO neurons, a cross-correlation analysis of the immunofluorescence localization of SV markers versus the localization of the respective AZ marker was performed (for details, see Materials and Methods). Thelongitudinalbut not the transversal-SD of the resulting ellipsoidal Gaussian surface was significantly increased in TKO neurons, indicating higher dispersion of SVs along the major axis of the axon (Fig. 2a). Dispersion affected to the same extent both excitatory and inhibitory terminals, as assayed by VGLUT1/Bassoon, VGAT/ Bassoon, and Syt/Piccolo double labeling (Fig. 2b).

\section{Dispersion of SV proteins occurs in vesicular compartments}

We next sought to determine whether mislocalized SVs were actually SVs per-

$\leftarrow$

clustering (MNND). All results are expressed as mean \pm SEM; ${ }^{* *} p<0.01$, Student's $t$ test. The number of synapses analyzed was 42 and 48 for WT and TK0, respectively. Scale bar, $200 \mathrm{~nm}$. $\boldsymbol{b}, 3 \mathrm{D}$ reconstruction of synapses from serial sections of WT and TKO neurons and morphometric analysis of synaptic and extrasynaptic vesicles. Top, Representative EM images of the serial sections used for the reconstruction (the analyzed neuron is highlighted in green). Middle, 3D visualization of SV distribution in the reconstructed synapse and the surrounding axon (green, plasma membrane; blue, SVs; red, AZ). To facilitate the visualization of SV dispersion, only SVs and AZs are shown in the bottom images. Bottom, Quantification of SV dispersion: the density of SVs present in the axonal region, the ratio between synaptic and extrasynaptic SVs, and the ratio between synaptic and axonal areas are reported. All results are expressed as mean \pm SEM; ${ }^{* *} p<0.01$, Student's $t$ test. The total number of reconstructed synapses was 10 and 12 for WT and TKO, respectively. Scale bars, $500 \mathrm{~nm}$. c, Distribution of recycled SVs in WT and TKO neurons. Left, EM images of WT and TKO synapses showing the distribution of SVs loaded with HRP (arrowheads point to HRP-positive SVs in the axon). Right, Box plot (minimum/maximum) of the distance of synaptic HRP-positive SVs from the AZ in WT and TKO synapses. Scale bars, $250 \mathrm{~nm}$. 
a
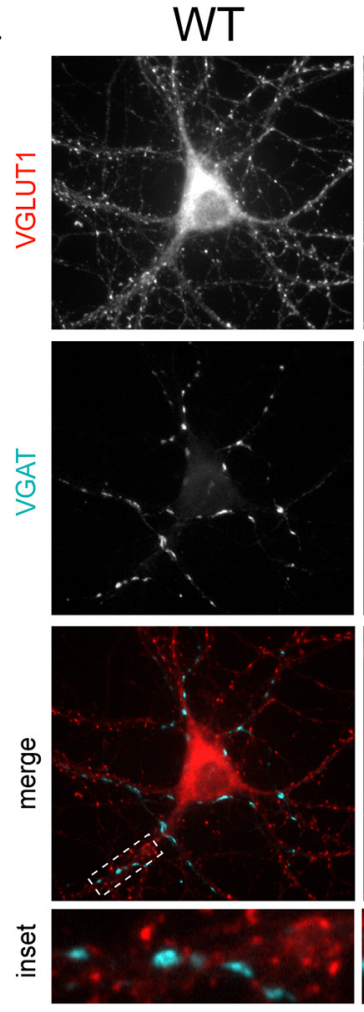

TKO
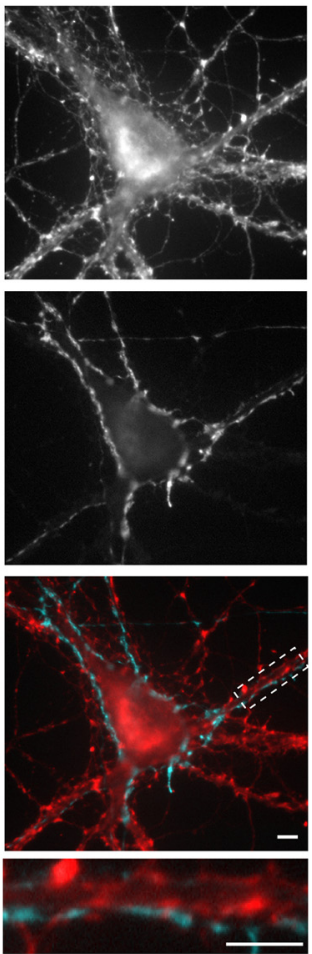

b
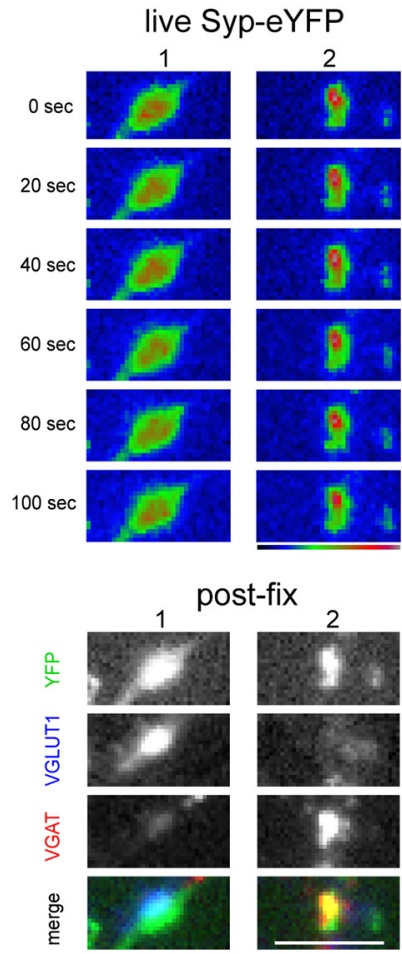
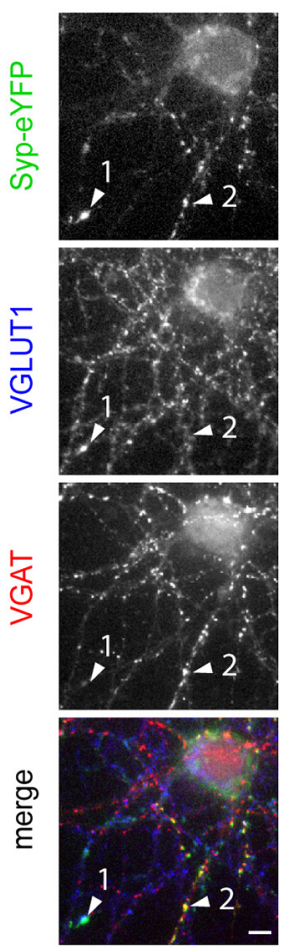

C

Syp-eYFP fluorescence
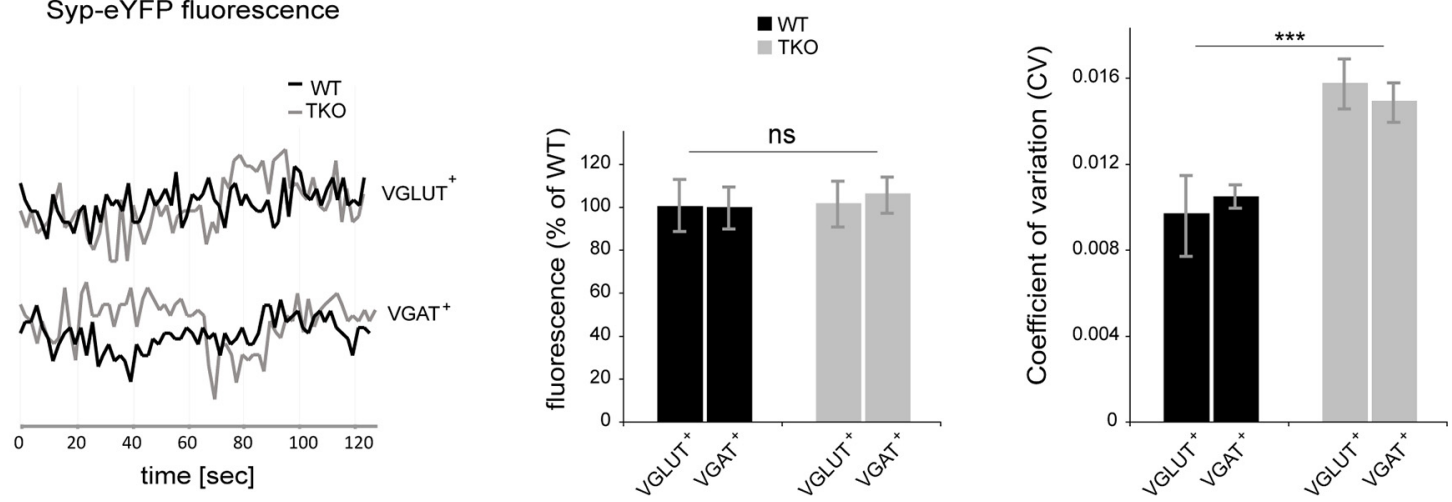

Figure 5. TKO neurons display enhanced SV dynamics at both excitatory and inhibitory synapses. $\boldsymbol{a}$, Low-magnification immunofluorescence images showing that both excitatory (VGLUT1 positive) and inhibitory (VGAT positive) SVs appear dispersed in TKO neurons but not in WT neurons. Insets, High-magnification images of the portions of neurites indicated by the rectangles. $\boldsymbol{b}$, Left, Vesicle dynamics was estimated through live imaging of single synapses of hippocampal neurons infected with Syp- eYFP. The fluorescence intensity of Syp-eYFP-positive puncta was monitored over a period of $2 \mathrm{~min}$ (live Syp-eYFP). Post hoc fixation and staining were performed to identify excitatory and inhibitory terminals (post-fix). Right, Low-magnification overview. Representative excitatory (1) and inhibitory (2) puncta are indicated by the arrowheads. c, Left, Representative traces of the temporal variation of fluorescence intensity at excitatory and inhibitory puncta (respectively, VGLUT1 ${ }^{+}$and VGAT ${ }^{+}$) in WT and TKO neurons. Middle and right, quantification of average fluorescence intensity (middle) and CV (CV = SD/average, right) of the synapses analyzed. The average bleach-corrected fluorescence is reported as mean \pm SEM; ${ }^{* * *} p<0.001$, WT vs TKO ( $>70$ boutons per condition; two-way ANOVA followed by Tukey's multiple comparison test). Scale bars, $5 \mu \mathrm{m}$.

manently fused with the axolemma, possibly because of impairment in SV cycling at the plasma membrane. For this purpose, we took advantage of the fluorescent reporter of SV cycling SpH to evaluate the fraction of SV components exposed to the extracellular surface and estimate the localization of acidic intracellular organelles (Miesenböck et al., 1998; Sankaranarayanan and Ryan, 2000). SpH experiences a 20.7 -fold increase of fluorescence when pH shifts from 5.5 to 7.5 (Miesenböck et al., 1998). Because the lumen of SVs is acidic ( $\mathrm{pH} \sim 5.5)$, the fluorescence of $\mathrm{SpH}$ in intracellular organelles is virtually absent in resting neurons. Brief exposure to $50 \mathrm{mM} \mathrm{NH}_{4} \mathrm{Cl}$ alkalinizes vesicles and visualizes all SpH molecules (Fig. 3a). The levels of expression (as deter- mined by field fluorescence after the application of $\left.\mathrm{NH}_{4} \mathrm{Cl}\right)$ and the surface fluorescence of $\mathrm{SpH}$ at synaptic boutons (calculated according to Sankaranarayanan and Ryan, 2000) were similar in WT and TKO neurons (Fig. 3b,c), indicating that a direct quantitative comparison of results from WT and TKO neurons was feasible and that SV protein delocalization was not attributable to aberrant exposure of SV proteins on the axolemma. Similar measurements at extrasynaptic sites were less reliable because of the much lower signal and the deriving high variability but are consistent nonetheless (data not shown). In the same experimental setup, we analyzed immobile puncta ( $>4 \mathrm{~min})$, undergoing a change in fluorescence intensity at the level of axons during 
a

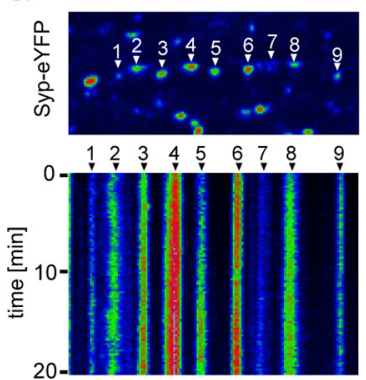

b
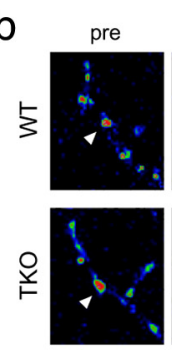

WT

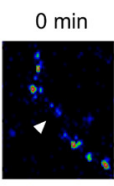

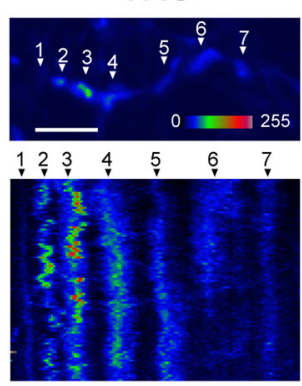
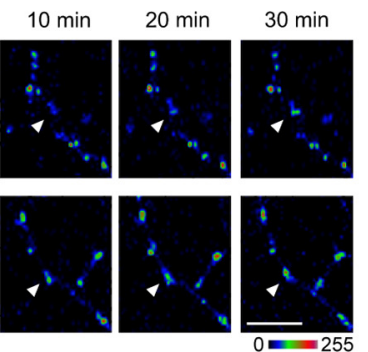

C

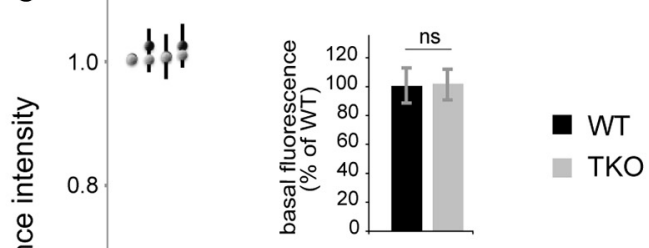

Figure 6. SVs acquire higher mobility at hippocampal synapses lacking Syns. $\boldsymbol{a}$, Live imaging of hippocampal neurons infected with Syp- eYFP. Top, Pseudocolored confocal images of presynaptic boutons overexpressing Syp-eYFP. White arrowheads indicate contacts followed over time. Bottom, Corresponding kymographs recorded over a 20 min period. $\boldsymbol{b}$, FRAP analysis for representative examples of slow-recovery bleaching experiments. White arrowheads indicate bleached synapses. c, Quantitative analysis of FRAP. To optimize resolution, separate experiments were performed for fast or slow recovery. The curves from the two conditions are shown (slow timescale: $n>3$ independent preparations, $>50$ boutons per condition; fast timescale: $n>3$ independent preparations, $>10$ boutons per condition; means \pm SEM). Recovery was consistently higher in TKO (point-by-point Mann-Whitney-Wilcoxon comparison vs WT, all $p<0.01$ for the slow recovery). The time constants of recovery estimated by double-exponential fitting of the average experimental curves are as follows: $\mathrm{WT}_{\text {fast }}=1.5 \pm 0.50 \mathrm{~min}, \mathrm{WT}_{\text {slow }}=28.0 \pm 12.7 \mathrm{~min}, \mathrm{TKO}_{\text {fast }}=1.1 \pm 0.33$ min, $\mathrm{TKO}_{\text {slow }}=34.9 \pm 11.6 \mathrm{~min}$. Inset, Average prebleaching fluorescence of the analyzed puncta. Scale bars, $5 \mu \mathrm{m}$.

$\mathrm{NH}_{4} \mathrm{Cl}$ alkalinization (for details, see Materials and Methods). Using this procedure, we estimated the levels of SVs in the bouton and in the adjacent extrasynaptic region of the axon (Fig. $3 a$, inset). This analysis showed a lower number of acidic vesicles at synapses and, conversely, an increased fraction of acidic vesicles in the extrasynaptic regions of TKO synaptic boutons, suggesting that TKO terminals have a different distribution of acidic vesicles (most likely SVs) at presynaptic AZs (Fig. 3d,e).

To test whether the aberrant localization of SVs impacts synapse functioning, we investigated the dynamics of SV recycling in our cultures. In the above experiments, a possible misplacement of the target protein $(\mathrm{SpH})$ from SV membranes might have made our approach less sensitive, partly masking the differences between the two genotypes. To more reliably address SV membrane, in the following experiments, we transduced neurons with a lentivirus encoding SypHy, a $\mathrm{pH}$-sensitive chimeric SV protein that is targeted to SVs more efficiently with respect to $\mathrm{SpH}$ (Granseth et al., 2006). The efficient expression provided by lentiviruses, coupled with automated analysis, reduces the artifacts attributable to single-cell overexpression and manual selection of ROIs. Hippocampal neurons were infected at 10 DIV and exposed at $>15$ DIV to two field stimulation protocols, 40 APs at 20 $\mathrm{Hz}$ or $300 \mathrm{APs}$ at $10 \mathrm{~Hz}$, to estimate the release from either the "readily releasable" or the "recycling" pool of SVs, respectively (Burrone et al., 2006). After each experimental session, the total repertoire of vesicles was verified by $\mathrm{NH}_{4} \mathrm{Cl}$ alkalinization. The surface fluorescence and the initial fluorescence $\left(F_{0}\right)$ at boutons were comparable (Fig. $3 f$ ). The short stimulation protocol revealed no changes in the size of the SV pool that readily contributes to synaptic transmission (Fig. $3 g$ ), in accordance with the observation that TKO synapses have no change in docked SVs (Gitler et al., 2004; Siksou et al., 2007). In contrast, a prolonged stimulation that challenges SV cycling and trafficking between pools revealed a clear defect in TKO synaptic boutons, suggesting that SV delocalization primarily affects recycling SVs (Fig. 3h). The total amount of SypHy acidic vesicles was also significantly reduced in TKO terminals (Fig. 3i), consistent with the data obtained with $\mathrm{SpH}$ and EM (see below).

\section{EM confirms dispersion of bona fide SVs within the axonal lumen of TKO neurons}

The dispersion phenotype observed in our cultures was further investigated by EM. As reported previously (Gitler et al., 2004; Siksou et al., 2007), in synapses of neurons lacking Syns, SVs were significantly diminished with respect to WT synapses and showed a substantial change in distribution (Fig. 4a). Similarly to WT, TKO terminals exhibited a cluster of SV close to the AZ and a population of dispersed vesicles farther away from it (Fig. 4a,c). However, whereas in the closest proximity $(50 \mathrm{~nm})$ of the AZ the amount of SVs was similar in the two genotypes, the SV number dramatically dropped at increasing distances from the AZ in TKO terminals. In accordance with this finding, the analysis of SV spatial distribution in TKO neurons showed an increase in the MNND between SVs and a decrease in the percentage of SVs present in a cluster, considered as a group of at least eight SVs no more that $100 \mathrm{~nm}$ far apart $(39.6 \pm 16.1 \%$ in WT vs $7.8 \pm 20.1 \%$ in TKO), clearly showing that SVs are less efficiently packed in terminals lacking Syns. The average number of docked vesicles was unaffected (Fig. 4a). In contrast, we did not observe either changes in average SV and endosome diameters or abnormal endocytic intermediates. Altogether, these observations indicate that, at the terminal, Syns contribute to the maintenance of the presynaptic cluster of SVs starting from a minimum distance of $100 \mathrm{~nm}$ apart from the $\mathrm{AZ}$, as reported previously (Pieribone et al., 1995; Gitler et al., 2004; Tao-Cheng, 2006; Siksou et al., 2007). 
To understand whether SV unclustering at synapses could contribute to redistribution within axons, 3D reconstruction and quantification from EM serial sections were performed (Fig. 4b). As suggested by the presence of $\mathrm{SpH}$ acidic vesicles in the perisynaptic region, we found that TKO synapses have a significantly increased number of SVs localized in the axonal shaft. Consistently, the ratio of SVs at synapses versus SVs within axons was greatly decreased in TKO neurons, in the absence of a difference in the relative area of the synaptic and the axonal compartments. These results suggest that a substantial percentage of SVs are dispersed along the axon in TKO neurons.

To get additional insight into the origin of the dispersed SVs, we acutely stimulated neurons in the presence of HRP. After stimulation with 900 APs at $20 \mathrm{~Hz}$, numerous labeled SVs (corresponding to SVs that had undergone recycling during the stimulation period) were visualized in terminals of both genotypes (Fig. 4c). Box plot analysis of location of HRP-positive SVs suggested a difference in distribution similar to that described above for resting vesicles. Moreover, in both genotypes, occasional HRP-positive SVs were visualized in axonal shafts.

Syns control the dynamic properties and mobilization of SVs at both excitatory and inhibitory terminals

Because the labeling of TKO fixed cells suggests that dispersion occurs in both excitatory and inhibitory neurons (Figs. $2 b, 5 a$ ), we decided to study the dynamic properties of SVs in more detail. For this purpose, we performed live imaging experiments of neurons infected with a lentivirus encoding for the SV protein Syp tagged with eYFP on the cytosolic tail. Single boutons were imaged for a 2 min period, and fluorescence fluctuations in the respective ROIs were monitored over time (an example is shown in Fig. 5b). After imaging, excitatory and inhibitory synapses were retrospectively identified by post hoc fixation and immunolabeling for VGLUT1 and VGAT (Fig. 5b, right side). We observed that, although the average fluorescence intensity was similar in WT and TKO synapses, the average CV (a measure of fluorescence variation with time in the corresponding ROI) was significantly increased at both VGLUT1- and VGAT-positive terminals of TKO boutons (Fig. 5c). The higher variability with time, at both excitatory and inhibitory synapses, points to a higher SV mobility in the absence of Syns.

In line with these observations, WT synapses infected with Syp-eYFP were characterized by a well-defined punctate pattern at the confocal microscope that was strikingly stable over time, as visualized in the kymograph, a graphical representation of spatial position over time (Fig. $6 a$, left). In contrast, puncta had less defined boundaries in TKO neurons and looked less stable over time (Fig. $6 a$, right).

To further estimate SV movement and cluster stability in SypeYFP-infected neurons, we used FRAP of single synaptic boutons (Fig. 6b). Bleaching apparently was more efficient in WT (87\%) than in TKO $(80 \%)$, possibly because of differences in vesicle movement during the procedure. The rate of recovery after bleaching was biexponential and displayed comparable kinetics in the two genotypes, with time constants $( \pm S D)$ of $1.5 \pm 0.50$ and $28 \pm 12.7 \mathrm{~min}$ for WT neurons and $1.1 \pm 0.33$ and $34.9 \pm$ $11.6 \mathrm{~min}$ for TKO neurons. The weights of the two exponential components, normalized to the initial fluorescence levels, were $18 \pm 2.6$ and $16 \pm 1.6 \%$ for WT neurons vs $28 \pm 3.9$ and $31 \pm$ $2.2 \%$ for TKO neurons. This demonstrates that the respective extent of the fast and slow recovery components were similarly affected in TKO boutons and that TKO boutons exhibited an
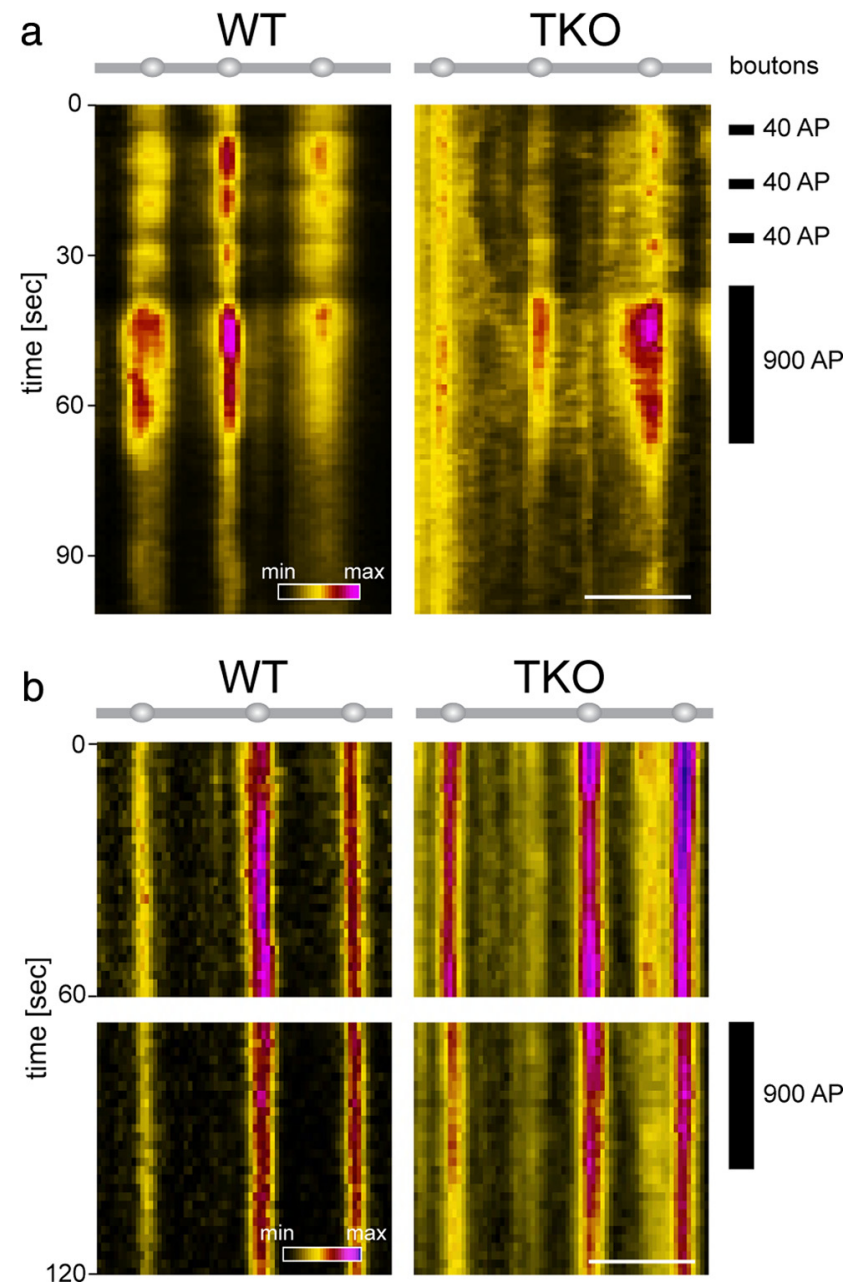

Figure 7. Electrical stimulation induces exocytosis of dispersed SVs in TKO neurons. Pseudocolored kymographs of synaptic boutons of hippocampal neurons infected with a lentivirus expressing either SypHy $(\boldsymbol{a})$ or Syp- eYFP $(\boldsymbol{b})$. Electrical field stimulation was used to stimulate activity. $\boldsymbol{a}$, Three trains of 40 APs at $20 \mathrm{~Hz}$ were followed by a train of $900 \mathrm{APs}$ at $20 \mathrm{~Hz}$, as indicated. SypHy fluorescence (data not normalized for bleaching) increases during stimulation, revealing exocytosed SVs. In TKO neurons, note the presence of fused SVs in intersynaptic regions, which is more apparent during prolonged stimulation. $\boldsymbol{b}$, Stimulation of terminals expressing an inert fluorescent SV tag (Syp- eYFP) indicates that acute depolarization does not change the localization of SVs, which are already delocalized in TKO neurons. Scale bars, $5 \mu \mathrm{m}$.

asymptotic recovery to $79 \%$ of the initial fluorescence $(65 \pm 6.9 \%$ at $40 \mathrm{~min}$ ) versus $\sim 47 \%$ in WT boutons ( $41 \pm 5.1 \%$ at $40 \mathrm{~min}$ ). These values indicate the presence of a larger fraction of mobile SVs in TKO; however, their mobility rates and their partition in two kinetically distinct pools $(\sim 50 \%$ ) were quite similar (Fig. $6 c$ ), suggesting that, although the TKO terminals contain less SVs (see above), a greater fraction thereof can be rapidly mobilized, with no apparent changes in the mechanisms of such mobilization, as deduced from the similar kinetics.

\section{Dispersed SVs in presynaptic boutons lacking Syns are exocytosis competent}

The relationships between SV dispersion and neuronal activity were assessed in neurons infected with SypHy and followed in time-lapse imaging during the application of electrical field stimulation. In the kymographs of SypHy fluorescence emission (Fig. $7 a$ ), the two genotypes displayed a distinct spatial distribution of SV exocytosis. In WT neurons, electrical stimulation induced an 
a
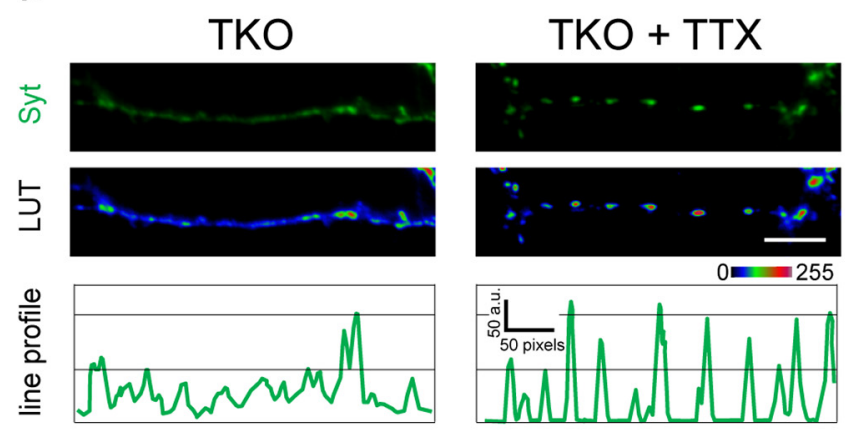

C

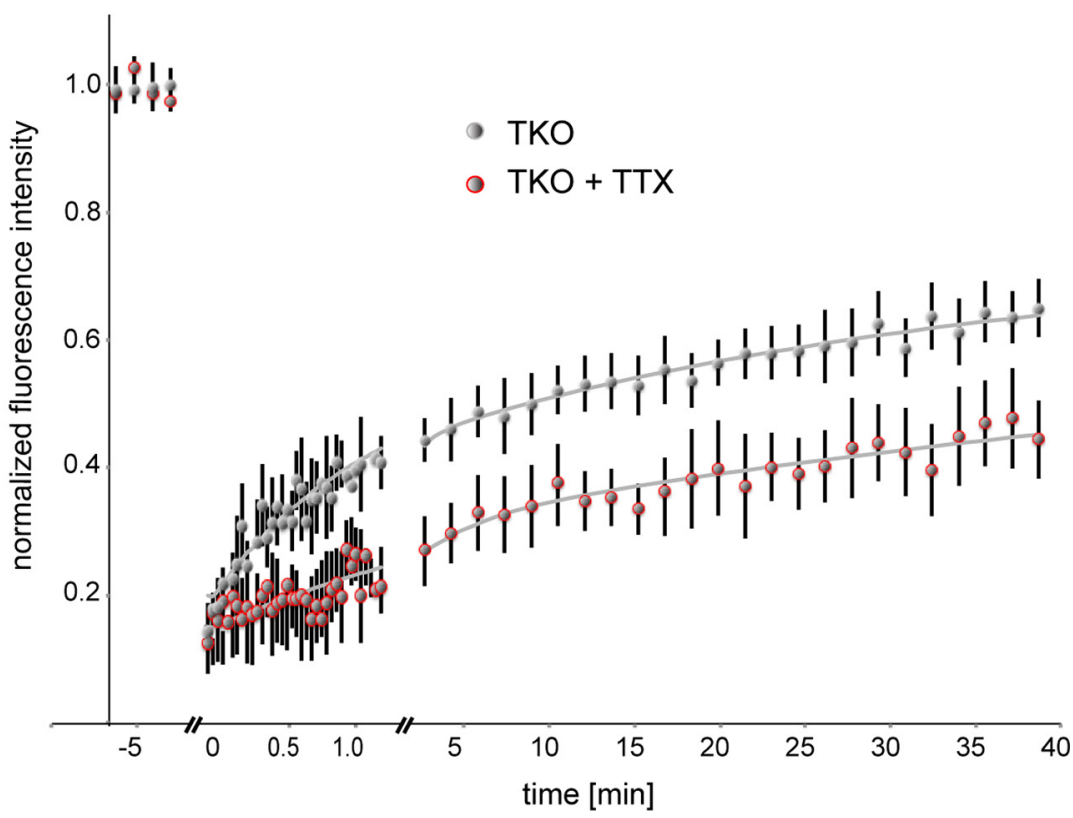

Figure 8. Chronic blockade of electrical activity rescues SV protein dispersion and mobility in neurons lacking Syns. $\boldsymbol{a}$, Isolated axonal tracts showing the distribution pattern of Syt by immunofluorescence microscopy in TKO neurons either untreated or after chronic activity blockade by application of TTX for $48 \mathrm{~h}$. The corresponding lookup table (LUT) and the fluorescence intensity line profiles are reported under each image to appreciate longitudinal dispersion. Scale bar, $5 \mu \mathrm{m}$. $\boldsymbol{b}$, Statistical evaluation of SV dispersion by longitudinal cross-correlation for the endogenous SV marker Syt with the presynaptic scaffolding protein Piccolo ( $n=3$ independent experiments; $>40$ boutons per condition, mean $\pm S E M ;{ }^{* *} p<0.01$, Student's $t$ test vs untreated). c, FRAP analysis for the evaluation of SV mobility in Syp-eYFP-infected TKO neurons either untreated (gray symbols) or after treatment with TTX for $48 \mathrm{~h}$ (red symbols). Experiments were performed as described in the legend to Figure 6 (slow timescale: $n>3$ independent preparations, $>50$ boutons per condition; fast timescale: $n>3$ independent preparations, $>10$ boutons per condition, means \pm SEM). TKO data from Figure 8 are reported here for comparison. Recovery was consistently lower in the TKO neurons after TTX treatment (point-by-point Mann-Whitney-Wilcoxon comparison vs TTX, all $p<0.01$ for the slow recovery). The time constants of recovery estimated by double-exponential fitting of the merged curves are as follows: TKO $_{\text {fast }}=1.1 \pm 0.33$ $\min , \mathrm{TKO}_{\text {slow }}=34.9 \pm 11.6 \mathrm{~min}, \mathrm{TKO}+\mathrm{TTX}_{\text {fast }}=2.5 \pm 1.56 \mathrm{~min}, \mathrm{TKO}+\mathrm{TTX}_{\text {slow }}=47.5 \pm 22.1 \mathrm{~min}$.

increase in fluorescence proportional to the intensity of stimulation and was highly localized at the responding boutons. In contrast, as also suggested by the distribution of HRP-positive SVs in EM specimens, TKO boutons had a less organized distribution and fluorescence widely spread over the adjacent axonal tracts. Kymograph analysis of a similar experiment performed in neurons expressing Syp-eYFP (which does not undergo changes in fluorescence during exocytosis) showed that neuronal activity in the TKO neurons did not change the localization of SVs, which were already diffused before stimulation (Fig. $7 b$ ). Consistently, the blockade of synaptic network activity by acute application of the $\mathrm{Na}^{+}$channel-blocker TTX did not induce variations in SV distribution (data not shown).
Chronic blockade of activity rescues SV dispersion in neurons lacking Syns

The possibility that long-term variations in the levels of network activity influence SV distribution and trafficking in TKO neurons was tested by the application of TTX for $48 \mathrm{~h}$. Immunofluorescence staining for the SV protein Syt on isolated axonal tracts showed that, in TTX-treated TKO neurons, SVs efficiently reclustered at synaptic boutons (Fig. 8a). Crosscorrelation analysis showed that chronic TTX treatment decreased the SD of the longitudinal cross-correlated images, confirming the rescue of SV protein dispersion (Fig. 8b).

We next tested the dynamic properties of SVs under the same conditions, by measuring fluorescence recovery in FRAP experiments on Syp-eYFP-infected neurons pretreated for $48 \mathrm{~h}$ with TTX. In TTX-treated TKO neurons, SV mobilization was significantly decreased with respect to untreated neurons: bleaching eliminated $84 \%$ of the initial fluorescence. Also in this case, the kinetics of recovery was not significantly affected, with time constants $( \pm S D)$ of $2.5 \pm 1.56$ and $47.5 \pm 22.1 \mathrm{~min}$ for TTX-treated neurons versus $1.1 \pm 0.33$ and $34.9 \pm$ $11.6 \mathrm{~min}$ for untreated ones; the weights of the two exponentials $(18 \pm 3.4$ and $27 \pm 2.4 \%$ in TTX-treated neurons vs $28 \pm 3.9$ and $31 \pm 2.2 \%$ in untreated ones) were in the same range. However, the rapidly equilibrating fraction appeared to be decreased in treated neurons, as was the asymptotic recovery (61 vs $79 \%$, with $44 \pm 6.9$ vs $65 \pm 6.9 \%$ recovery at $40 \mathrm{~min}$; Fig. $8 \mathrm{c}$ ). In this case as well, as discussed for TKO versus WT neurons, the changes affected the fraction of SVs that can be mobilized (especially the rapidly mobilized ones) rather than the kinetics of the process. Nonetheless, SV mobilization was fully comparable with WT neurons treated with TTX (data not shown).

Thus, we analyzed the effects of the suppression of network activity in TKO neurons on the ultrastructure of nerve terminals (Fig. 9, top and middle). Notably, the ratio of synaptic versus axonal SVs was increased in both TKO and WT neurons treated with TTX (Fig. 9, bottom). The effect was particularly marked in TKO neurons, whose axonal SV density after TTX was not significantly different from that of WT neurons either untreated or chronically treated with TTX, in the absence of any change in the ratio between synaptic and axonal areas.

Finally, we investigated the dynamics of SV recycling in cultures treated for $48 \mathrm{~h}$ with TTX. During a brief ( $5 \mathrm{~min}$ ) washout of the drug, the surface fluorescence and the readily releasable and the recycling pools of SVs were not significantly different from those of WT neurons, whereas the total number 
of SVs showed a tendency to be diminished, although not significantly (data not shown). This suggests that prolonged TTX blockade could functionally attenuate the differences caused by the absence of Syns.

\section{Discussion}

In accordance with previous ultrastructural studies (Li et al., 1995; Rosahl et al., 1995; Takei et al., 1995; Siksou et al., 2007), the absence of Syns does not affect the distribution of presynaptic and postsynaptic scaffold proteins at synaptic contacts. In contrast, the presynaptic compartment of Syn TKO mice displays an altered SV clustering pattern with dispersion of SVs within the axonal lumen in both excitatory and inhibitory terminals, indicating that Syns are important not only for the correct targeting of SVs at presynaptic sites but also for the retention of SVs at boutons by preventing lateral diffusion.

In principle, two distinct mechanisms might disperse SVs in TKO neurons: either SVs diffuse more rapidly (increased mobility) or a mechanism of SV localization at synapses is lacking or impaired. FRAP experiments indicate that the rate of fluorescence recovery at bleached synapses is unaffected in TKO neurons, although these terminals recover their fluorescence to a markedly higher extent. This suggests that bleached and naive SVs exchange at a comparable rate (similar mobility) in the two genotypes, but a lower fraction of SVs exchanges at WT terminals, which points to the presence of a retention mechanism (presumably Syn binding) in WT, but not TKO, terminals. These data show that the basic properties of axonal transport in adult neurons are not regulated by Syns, whereas the socalled "superpool" of SVs, which is spanning several en passant boutons of the same axon (Denker and Rizzoli, 2010; Staras et al., 2010), is increased in TKO neurons. Very recent findings render this result particularly relevant because SV redistribution within the same axon might be essential for the competitive strengthening/weakening of presynaptic efficacy and might improve pattern discrimination in dendritic networks (Herzog et al., 2011; Padamsey and Jeans, 2012). Through the mobilization/immobilization of SVs, Syns might locally regulate the plastic properties at single presynapses both acting as restrictors, by contributing with a resilient force to the tenacity of the terminal (Fisher-Lavie et al., 2011) and serving as integrators of presynaptic signaling in a phosphorylation-dependent manner (Cesca et al., 2010).

The sizes of the SV pools determined from the SypHy signal after sustained electrical stimulation indicate that the SV pool, which can be mobilized by sustained stimulation ("recycling" pool), is significantly reduced in TKO neurons. These changes help explain the previously reported increased fatigability of synapses that lack Syns during prolonged stimulation (Terada et al., 1999; Gitler et al., 2004; Akbergenova and Bykhovskaia, 2007; Baldelli et al., 2007; Farisello et al., 2012).

Kymographic analysis shows that acute stimulation does not increase overall SV dispersion within a short timeframe in TKO neurons. These data are in accordance with the finding that electrical stimulation has no influence on the mobility of single SVs in short timeframes (Kamin et al., 2010), although a more careful evaluation with longer timeframes and/or stimulation protocols might be required, because this issue is still debated (Fisher-Lavie et al., 2011). Nevertheless, our results indicate that the population of delocalized SVs observed in TKO synapses includes exocytosis-competent SVs. Thus, it is possible that delocalized SV exocytosis and "off-target" release of transmitter occur; however, we cannot exclude that our observation simply reflects rapid diffusion of recycled vesicles (or of their membrane). The time course of SypHy fluorescence diffusion in kymographic recordings might suggest that SVs become alkalinized at the synaptic bouton and only later does their membrane diffuse (possibly after SVs have been 
reconstituted by endocytosis). However, additional experiments will be needed to properly address this aspect.

Although some studies have identified presynaptic components that regulate the maintenance of SVs at AZs (Bamji et al., 2003; Weimer et al., 2006; Lee et al., 2008; Mukherjee et al., 2010), a general consensus concerning the molecular actor(s) that localize SVs at synapses was missing (Gaffield and Betz, 2007; Siksou et al., 2007). Among the likely candidates contributing to SV spatial targeting and clustering, Syns are by far the most extensively studied. Ablation of Syns in mice leads to several defects, including a reduction in the number and an altered distribution of SVs in the presynaptic boutons (Rosahl et al., 1995; Takei et al., 1995; Ryan et al., 1996; Gitler et al., 2004; Siksou et al., 2007) that has been attributed to their function as SV tethering molecules.

Our work, together with a recently published paper (Orenbuch et al., 2012), are the first to directly address the redistribution of SVs at synaptic boutons and in the rest of the axon in the absence of Syns. Strikingly, both works establish that Syns are essential to shape presynaptic terminals, by maintaining compact SV clusters and by restricting SVs at the terminal. Previous experiments at the neuromuscular junction showed that, in the absence of Syns, recycling SVs are still targeted at terminals and SV mobility is not altered (Gaffield and Betz, 2007). However, the organization of SVs at motor terminals is profoundly different from that of en passant presynaptic terminals in the CNS (Rizzoli and Betz, 2005).

Concerning the role of the Syns in regulating the various SV pools, we show that, in TKO neurons, the total amount of recycling and resting SVs are decreased, in line with the hypothesis that Syns contribute to the number of vesicles that are available for exocytosis under relatively sustained stimulation. As suggested by Orenbuch et al. (2012), it is possible that the ratio of the recycling pool of SVs with respect to total SVs is regulated in a Syn-independent manner, because in their experiments WT and TKO terminals exchange the same fraction of recycling vesicles. However, it should be noted that those measurements were performed on neurons loaded with FM1-43 [N-(3-triethylammoniumpropyl)-4-(4-(dibutylamino)styryl) pyridinium dibromide] by exposure to a hyperkalemic medium, i.e., a stimulation that does not reproduce what happens in physiological conditions.

To further examine the possible influence of activity on SV localization, experiments were performed after $48 \mathrm{~h}$ incubation in TTX. This treatment proved able to revert the phenotype of TKO neurons, producing relocalization of SVs at boutons. Thus, it is possible that the diffused pattern of SVs observed in TKO terminals requires activity. The spontaneous activity of hippocampal neuron networks, which is already present at the stage of development of our experiments (Segal, 1983; our unpublished observations), might be responsible for the SV delocalization observed in unstimulated terminals.

It is noteworthy that acute TTX block does not revert the dispersion phenotype of TKO terminals, although it has been reported to decrease SV motion (Kamin et al., 2010). The most obvious explanation for this observation is that the mechanism(s) constraining SVs at nerve terminals in the absence of Syns has a low efficiency and requires a long time to revert the altered phenotype. Indeed, it has been proposed that recently endocytosed SVs are mobile and that they subsequently mature into "cluster-integrated" SVs (Denker and Rizzoli, 2010; Kamin et al., 2010). This would explain the presence of HRP-positive SVs in the axonal shaft in both genotypes.
We cannot exclude that the effects observed after $48 \mathrm{~h}$ treatment with TTX might also involve compensatory adaptation through modifications of gene expression and protein synthesis (Murthy et al., 2001). Indeed, adaptations to global changes in activity levels have been reported to occur both in vivo and in vitro through compensatory modifications in presynaptic and postsynaptic parameters of synaptic transmission (Turrigiano et al., 1998; Echegoyen et al., 2007; Cingolani et al., 2008). Together, our results suggest that SV retention at presynaptic sites is a robust process with a complex regulation comprising at least two mechanisms. One mechanism is strictly Syn dependent and temporally couples SV mobilization to neuronal activity. In the absence of Syns, an additional retention mechanism becomes apparent. This mechanism may not be in itself efficient enough to keep SVs at the synapse in the presence of spontaneous activity in TKO neurons but can be uncovered by prolonged activity blockade. Indeed, electron tomography of presynaptic terminals revealed the presence of small filaments linking SVs together (Siksou et al., 2007). Their persistence in TKO mice possibly provides a basis for an Syn-independent mechanism of SV clustering.

It is known that Syn ablation differentially affects neurotransmitter release in excitatory versus inhibitory neurons (Rosahl et al., 1995; Ryan et al., 1996; Terada et al., 1999; Gitler et al., 2004; Baldelli et al., 2007; Chiappalone et al., 2009; Farisello et al., 2012). In particular, excitatory transmission appears unaffected or even enhanced, whereas inhibitory transmission is impaired (Gitler et al., 2004; Chiappalone et al., 2009; Farisello et al., 2012). Interestingly, SV dispersion and increased mobility appears to occur in both GABAergic and glutamatergic terminals of TKO neurons. However, because of the different structural and functional features of the terminals, dispersion might lead to dissimilar consequences on neurotransmission: the relative SV depletion might impair release in highfrequency-firing GABAergic interneurons, whereas glutamatergic terminals might be less affected or even experience a less controlled release of dispersed SVs.

\section{References}

Akbergenova Y, Bykhovskaia M (2007) Synapsin maintains the reserve vesicle pool and spatial segregation of the recycling pool in drosophila presynaptic boutons. Brain Res 1178:52-64.

Amendola M, Venneri MA, Biffi A, Vigna E, Naldini L (2005) Coordinate dual-gene transgenesis by lentiviral vectors carrying synthetic bidirectional promoters. Nat Biotechnol 23:108-116.

Baldelli P, Fassio A, Valtorta F, Benfenati F (2007) Lack of synapsin I reduces the readily releasable pool of synaptic vesicles at central inhibitory synapses. J Neurosci 27:13520-13531.

Bamji SX, Shimazu K, Kimes N, Huelsken J, Birchmeier W, Lu B, Reichardt LF (2003) Role of beta-catenin in synaptic vesicle localization and presynaptic assembly. Neuron 40:719-731.

Banker GA, Cowan WM (1977) Rat hippocampal neurons in dispersed cell culture. Brain Res 126:397-342.

Benfenati F, Valtorta F, Rossi MC, Onofri F, Sihra T, Greengard P (1993) Interactions of synapsin I with phospholipids: Possible role in synaptic vesicle clustering and in the maintenance of bilayer structures. J Cell Biol 123:1845-1855.

Boido D, Farisello P, Cesca F, Ferrea E, Valtorta F, Benfenati F, Baldelli P (2010) Cortico-hippocampal hyperexcitability in synapsin I/II/III knockout mice: age-dependency and response to the antiepileptic drug levetiracetam. Neuroscience 171:268-283.

Bonanomi D, Menegon A, Miccio A, Ferrari G, Corradi A, Kao HT, Benfenati F, Valtorta F (2005) Phosphorylation of synapsin I by cAMP-dependent protein kinase controls synaptic vesicle dynamics in developing neurons. J Neurosci 25:7299-7308.

Burrone J, Li Z, Murthy VN (2006) Studying vesicle cycling in presynaptic 
terminals using the genetically encoded probe synaptopHluorin. Nat Protoc 1:2970-2978.

Ceccaldi PE, Grohovaz F, Benfenati F, Chieregatti E, Greengard P, Valtorta F (1995) Dephosphorylated synapsin I anchors synaptic vesicles to actin cytoskeleton: an analysis by videomicroscopy. J Cell Biol 128:905-912.

Cesca F, Baldelli P, Valtorta F, Benfenati F (2010) The synapsins: key actors of synapse function and plasticity. Prog Neurobiol 91:313-348.

Chiappalone M, Casagrande S, Tedesco M, Valtorta F, Baldelli P, Martinoia S, Benfenati F (2009) Opposite changes in glutamatergic and GABAergic transmission underlie the diffuse hyperexcitability of synapsin I-deficient cortical networks. Cereb Cortex 19:1422-1439.

Chi P, Greengard P, Ryan TA (2001) Synapsin dispersion and reclustering during synaptic activity. Nat Neurosci 4:1187-1193.

Chi P, Greengard P, Ryan TA (2003) Synaptic vesicle mobilization is regulated by distinct synapsin I phosphorylation pathways at different frequencies. Neuron 38:69-78.

Cingolani LA, Thalhammer A, Yu LM, Catalano M, Ramos T, Colicos MA, Goda Y (2008) Activity-dependent regulation of synaptic AMPA receptor composition and abundance by beta3 integrins. Neuron 58:749-762.

Denker A, Rizzoli SO (2010) Synaptic vesicle pools: an update. Front Synaptic Neurosci 2:135.

Echegoyen J, Neu A, Graber KD, Soltesz I (2007) Homeostatic plasticity studied using in vivo hippocampal activity-blockade: synaptic scaling, intrinsic plasticity and age-dependence. PLoS One 2:e700.

Farisello P, Boido D, Nieus T, Medrihan L, Cesca F, Valtorta F, Baldelli P, Benfenati F (2012) Synaptic and extrasynaptic origin of the excitation/ inhibition imbalance in the hippocampus of synapsin I/II/III knockout mice. Cereb Cortex. Advance online publication. Retrieved July 24, 2011. doi:10.1093/cercor/bhs041.

Fassio A, Merlo D, Mapelli J, Menegon A, Corradi A, Mete M, Zappettini S, Bonanno G, Valtorta F, D'Angelo E, Benfenati F (2006) The synapsin domain $\mathrm{E}$ accelerates the exoendocytotic cycle of synaptic vesicles in cerebellar Purkinje cells. J Cell Sci 119:4257-4268.

Fassio A, Patry L, Congia S, Onofri F, Piton A, Gauthier J, Pozzi D, Messa M, Defranchi E, Fadda M, Corradi A, Baldelli P, Lapointe L, St-Onge J, Meloche C, Mottron L, Valtorta F, Khoa Nguyen D, Rouleau GA, Benfenati F, Cossette P (2011) SYN1 loss-of-function mutations in autism and partial epilepsy cause impaired synaptic function. Hum Mol Genet 20:2297-2307.

Feng J, Chi P, Blanpied TA, Xu Y, Magarinos AM, Ferreira A, Takahashi RH, Kao HT, McEwen BS, Ryan TA, Augustine GJ, Greengard P (2002) Regulation of neurotransmitter release by synapsin III. J Neurosci 22:4372-4380.

Fisher-Lavie A, Zeidan A, Stern M, Garner CC, Ziv NE (2011) Use dependence of presynaptic tenacity. J Neurosci 31:16770-16780.

Gaffield MA, Betz WJ (2007) Synaptic vesicle mobility in mouse motor nerve terminals with and without synapsin. J Neurosci 27:13691-13700.

Garcia CC, Blair HJ, Seager M, Coulthard A, Tennant S, Buddles M, Curtis A, Goodship JA (2004) Identification of a mutation in synapsin I, a synaptic vesicle protein, in a family with epilepsy. J Med Genet 41:183-186.

Gitler D, Takagishi Y, Feng J, Ren Y, Rodriguiz RM, Wetsel WC, Greengard P, Augustine GJ (2004) Different presynaptic roles of synapsins at excitatory and inhibitory synapses. J Neurosci 24:11368-11380.

Granseth B, Odermatt B, Royle SJ, Lagnado L (2006) Clathrin-mediated endocytosis is the dominant mechanism of vesicle retrieval at hippocampal synapses. Neuron 51:773-786.

Herzog E, Nadrigny F, Silm K, Biesemann C, Helling I, Bersot T, Steffens H, Schwartzmann R, Nägerl UV, El Mestikawy S, Rhee J, Kirchhoff F, Brose $\mathrm{N}$ (2011) In vivo imaging of intersynaptic vesicle exchange using VGLUT1 venus knock-in mice. J Neurosci 31:15544-15559.

Hilfiker S, Schweizer FE, Kao HT, Czernik AJ, Greengard P, Augustine GJ (1998) Two sites of action for synapsin domain $E$ in regulating neurotransmitter release. Nat Neurosci 1:29-35.

Hilfiker S, Benfenati F, Doussau F, Nairn AC, Czernik AJ, Augustine GJ, Greengard P (2005) Structural domains involved in the regulation of transmitter release by synapsins. J Neurosci 25:2658-2669.

Humeau Y, Doussau F, Vitiello F, Greengard P, Benfenati F, Poulain B (2001) Synapsin controls both reserve and releasable synaptic vesicle pools during neuronal activity and short-term plasticity in Aplysia. J Neurosci 21:4195-4206.

Hvalby Ø, Jensen V, Kao HT, Walaas SI (2006) Synapsin-regulated synaptic transmission from readily releasable synaptic vesicles in excitatory hippocampal synapses in mice. J Physiol 571:75-82.

Kaech S, Banker G (2006) Culturing hippocampal neurons. Nat Protoc 1:2406-2415.

Kamin D, Lauterbach MA, Westphal V, Keller J, Schönle A, Hell SW, Rizzoli SO (2010) High- and low-mobility stages in the synaptic vesicle cycle. Biophys J 99:675-684.

Kao HT, Porton B, Hilfiker S, Stefani G, Pieribone VA, DeSalle R, Greengard P (1999) Molecular evolution of the synapsin gene family. J Exp Zool 285:360-377.

Ketzef M, Kahn J, Weissberg I, Becker AJ, Friedman A, Gitler D (2011) Compensatory network alterations upon onset of epilepsy in synapsin triple knock-out mice. Neuroscience 189:108-122.

Lee SH, Peng IF, Ng YG, Yanagisawa M, Bamji SX, Elia LP, Balsamo J, Lilien J, Anastasiadis PZ, Ullian EM, Reichardt LF (2008) Synapses are regulated by the cytoplasmic tyrosine kinase fer in a pathway mediated by p120catenin, fer, SHP-2, and beta-catenin. J Cell Biol 183:893-908

Li L, Chin LS, Shupliakov O, Brodin L, Sihra TS, Hvalby O, Jensen V, Zheng D, McNamara JO, Greengard P (1995) Impairment of synaptic vesicle clustering and of synaptic transmission, and increased seizure propensity, in synapsin I-deficient mice. Proc Natl Acad Sci U S A 92:9235-9239.

Miesenböck G, De Angelis DA, Rothman JE (1998) Visualizing secretion and synaptic transmission with $\mathrm{pH}$-sensitive green fluorescent proteins. Nature 394:192-195.

Mukherjee K, Yang X, Gerber SH, Kwon HB, Ho A, Castillo PE, Liu X, Südhof TC (2010) Piccolo and bassoon maintain synaptic vesicle clustering without directly participating in vesicle exocytosis. Proc Natl Acad Sci U S A 107:6504-6509.

Murthy VN, De Camilli P (2003) Cell biology of the presynaptic terminal. Annu Rev Neurosci 26:701-728.

Murthy VN, Schikorski T, Stevens CF, Zhu Y (2001) Inactivity produces increases in neurotransmitter release and synapse size. Neuron 32:673-682.

Orenbuch A, Shalev L, Marra V, Sinai I, Lavy Y, Kahn J, Burden JJ, Staras K, Gitler D (2012) Synapsin selectively controls the mobility of resting pool vesicles at hippocampal terminals. J Neurosci 32:3969-3980.

Padamsey Z, Jeans A (2012) Imaging synaptic vesicles using VGLUT1venus knock-in mice: insights into the dynamic nature of intersynaptic vesicle exchange. J Neurosci 32:3284-3286.

Pennuto M, Bonanomi D, Benfenati F, Valtorta F (2003) Synaptophysin I controls the targeting of VAMP2/synaptobrevin II to synaptic vesicles. Mol Biol Cell 14:4909-4919.

Phair RD, Gorski SA, Misteli T (2004) Measurement of dynamic protein binding to chromatin in vivo, using photobleaching microscopy. Methods Enzymol 375:393-414.

Pieribone VA, Shupliakov O, Brodin L, Hilfiker-Rothenfluh S, Czernik AJ, Greengard P (1995) Distinct pools of synaptic vesicles in neurotransmitter release. Nature 375:493-497.

Rizzoli SO, Betz WJ (2005) Synaptic vesicle pools. Nat Rev Neurosci 6:57-69.

Rosahl TW, Spillane D, Missler M, Herz J, Selig DK, Wolff JR, Hammer RE, Malenka RC, Südhof TC (1995) Essential functions of synapsins I and II in synaptic vesicle regulation. Nature 375:488-493.

Ryan TA, Li L, Chin LS, Greengard P, Smith SJ (1996) Synaptic vesicle recycling in synapsin I knock-out mice. J Cell Biol 134:1219-1227.

Sankaranarayanan S, Ryan TA (2000) Real-time measurements of vesicleSNARE recycling in synapses of the central nervous system. Nat Cell Biol 2:197-204.

Sankaranarayanan S, De Angelis D, Rothman JE, Ryan TA (2000) The use of pHluorins for optical measurements of presynaptic activity. Biophys J 79:2199-2208.

Segal M (1983) Rat hippocampal neurons in culture: responses to electrical and chemical stimuli. J Neurophysiol 50:1249-1264.

Siksou L, Rostaing P, Lechaire JP, Boudier T, Ohtsuka T, Fejtov á A, Kao HT, Greengard P, Gundelfinger ED, Triller A, Marty S (2007) Threedimensional architecture of presynaptic terminal cytomatrix. J Neurosci 27:6868-6877. 
Staras K, Branco T, Burden JJ, Pozo K, Darcy K, Marra V, Ratnayaka A, Goda Y (2010) A vesicle superpool spans multiple presynaptic terminals in hippocampal neurons. Neuron 66:37-44.

Sun J, Bronk P, Liu X, Han W, Südhof TC (2006) Synapsins regulate usedependent synaptic plasticity in the calyx of held by a $\mathrm{Ca}^{2+} /$ calmodulindependent pathway. Proc Natl Acad Sci U S A 103:2880-2885.

Takei Y, Harada A, Takeda S, Kobayashi K, Terada S, Noda T, Takahashi T, Hirokawa N (1995) Synapsin I deficiency results in the structural change in the presynaptic terminals in the murine nervous system. J Cell Biol 131:1789-1800.

Tao-Cheng JH (2006) Activity-related redistribution of presynaptic proteins at the active zone. Neuroscience 141:1217-1224.

Terada S, Tsuijimoto T, Takei Y, Takahashi T, Hirokawa N (1999) Impairment of inhibitory synaptic transmission in mice lacking synapsin I. J Cell Biol 145:1039-1048.
Turrigiano GG, Leslie KR, Desai NS, Rutherford LC, Nelson SB (1998) Activity-dependent scaling of quantal amplitude in neocortical neurons. Nature 391:892-896.

Valtorta F, Benfenati F (1995) Membrane trafficking in nerve terminals. Adv Pharmacol 32:505-557.

Valtorta F, Jahn R, Fesce R, Greengard P, Ceccarelli B (1988) Synaptophysin (p38) at the frog neuromuscular junction: Its incorporation into the axolemma and recycling after intense quantal secretion. J Cell Biol 107:2717-2727.

Valtorta F, Greengard P, Fesce R, Chieregatti E, Benfenati F (1992) Effects of the neuronal phosphoprotein synapsin I on actin polymerization. I. Evidence for a phosphorylation-dependent nucleating effect. J Biol Chem 267:11281-11288.

Weimer RM, Gracheva EO, Meyrignac O, Miller KG, Richmond JE, Bessereau JL (2006) UNC-13 and UNC-10/rim localize synaptic vesicles to specific membrane domains. J Neurosci 26:8040-8047. 